\title{
Towards an automated tool to evaluate the impact of the nuclear modification of the gluon density on quarkonium, $D$ and $B$ meson production in proton-nucleus collisions
}

\author{
Jean-Philippe Lansberg ${ }^{1, a}{ }^{(\mathbb{C}}$, Hua-Sheng Shao ${ }^{2}$ \\ ${ }^{1}$ IPNO, Université Paris-Saclay, Univ. Paris-Sud, CNRS/IN2P3, 91406 Orsay, France \\ 2 Theoretical Physics Department, CERN, 1211 Geneva 23, Switzerland
}

Received: 27 October 2016 / Accepted: 12 December 2016 / Published online: 27 December 2016

(C) The Author(s) 2016. This article is published with open access at Springerlink.com

\begin{abstract}
We propose a simple and model-independent procedure to account for the impact of the nuclear modification of the gluon density as encoded in nuclear collinear PDF sets on two-to-two partonic hard processes in protonnucleus collisions. This applies to a good approximation to quarkonium, $D$ and $B$ meson production, generically referred to $\mathcal{H}$. Our procedure consists in parametrising the square of the parton scattering amplitude, $\mathcal{A}_{g g \rightarrow \mathcal{H} X}$ and constraining it from the proton-proton data. Doing so, we have been able to compute the corresponding nuclear modification factors for $J / \psi, \Upsilon$ and $D^{0}$ as a function of $y$ and $P_{T}$ at $\sqrt{s_{\mathrm{NN}}}=5$ and $8 \mathrm{TeV}$ in the kinematics of the various LHC experiments in a model independent way. It is of course justified since the most important ingredient in such evaluations is the probability of each kinematical configuration. Our computations for $D$ mesons can also be extended to $B$ meson production. To further illustrate the potentiality of the tool, we provide - for the first time - predictions for the nuclear modification factor for $\eta_{c}$ production in $p \mathrm{~Pb}$ collisions at the LHC.
\end{abstract}

\section{Introduction}

For many years, open and closed heavy-flavour production in hadron-hadron, hadron-nucleus and nucleus-nucleus collisions has been a major subject of investigations, on both experimental and theoretical sides (see [1] for a review in the context of the first LHC results and [2-6] for earlier reviews). In addition of helping us to understand the interface between the perturbative and non-perturbative regimes of QCD in hadron-hadron collisions, these reactions are also sensitive to - and thus probe - the properties of the possible deconfined state of matter (QGP) resulting from nucleus-nucleus $(A A)$ collisions at ultra-relativistic energies.

\footnotetext{
a e-mail: lansberg@in2p3.fr
}

Yet, heavy-flavour production can also be affected by other nuclear effects ${ }^{1}$ which are not related to a phase transition; they should in principle be subtracted in a way or another to study the QGP. These are typically believed to be the only ones acting in proton/deuteron-nucleus $(p A)$ collisions at fixed-target, RHIC and LHC energies. Experimental results from RHIC and the LHC in $p A$ collisions [1] have shown that the yields and the spectra of $J / \psi, \Upsilon, D$ and $B$ are indeed modified in a magnitude which cannot simply be ignored in QGP studies. Many effects can be at play: break up within the nucleus $[7,8]$ or with comovers for the quarkonia [912], coherent or incoherent energy loss [13-17], colour filtering [18], saturation/small- $x /$ coherence effects [19-23], and the modification of the parton fluxes, as encoded in nuclear Parton Distribution Functions (nPDFs) [24-28].

In what follows, we will focus on the latter effects as a baseline for comparisons with experimental data. Our aim here is not to argue that it is indeed the dominant effect at RHIC and the LHC. Yet, a couple of recent comparisons $[1,29,30]$ have shown that the magnitude of the gluon modification in usual nPDF fits is in reasonnable agreement with quarkonium, $D$ and $B$ meson data in $p \mathrm{~Pb}$ collisions at the LHC.

nPDF fits are constantly updated with new data, ${ }^{2}$ recently from the LHC, and we have found it useful to propose a simple and model-independent procedure to account for the $\mathrm{nPDF}$ impact, in the particular case of gluon-induced $2 \rightarrow 2$

\footnotetext{
1 In what follows, we will call them "cold nuclear matter effects".

2 We would like to mention that none of the global proton PDF fitting groups has so far used the heavy-flavour data in proton-proton collisions. However, some progress has recently been made in this direction. For example, the cross-section ratios of open-heavy-flavour production data from LHCb have been used to constrain the small- $x$ gluon density in proton PDFs [31].
} 
reactions. ${ }^{3}$ Such a procedure, to be encoded in a user friendly forthcoming tool, would then allow anybody to make up one's mind about the typical expected magnitude of the gluon nuclear modifications on a given probe.

In the past, shortcut procedures using simplified kinematics (like the one of Drell-Yan at LO, that is $2 \rightarrow 1$ ) have widely been used [32-36]. However, it has been shown [3739 ] that it can yield to systematic differences and, in principle, it cannot account for the $P_{T}$ dependence of the yield. In general, it is just better to rely on a more proper $2 \rightarrow 2$ kinematics, although some higher QCD corrections could involve more than 2 hard particles in the final state at large $P_{T}$. For this purpose, a probabilistic Glauber Monte Carlo code, JIN [3739], dedicated to the quarkonium case, has been developed to account for the geometry of the nuclear collisions and the impact parameter dependence of the nuclear effects at play along with the nPDF effect with an exact kinematics. However, as for now, the code deals with a limited number of processes (including though $b$ production [40]) and of nPDFs; a simpler tool focusing on a $2 \rightarrow 2$ kinematics as the one we propose here is therefore very complementary. Eventually, both tools could interfaced or merged. Other tools, like APPLGRID [41] and FASTNLO [42], with a similar spirit also exist but for different observables.

As will be explained below, the tool which we propose is based on HELAC-ONIA $[43,44]$ (but is not restricted to quarkonia) can use any nPDF set included in the library LHAPDF5 [45,46] and LHAPDF6 [47] and does not rely on any model for the hard-probe production, but on $p p$ measurements which are used to tune the partonic-scattering elements, keeping in mind the limitation that it should be tuned for each process and in principle for possible different kinematical region with the chosen proton PDF and factorisation scale.

\section{Our approach}

As announced, our approach is based on a data-driven modelling of the scattering at the partonic level. Once folded with proton PDFs, they yield $p p$ cross sections and, when folded with one proton PDF and one nuclear PDF, they yield $p A$ cross sections. Such a choice is essentially motivated by the case of inclusive quarkonium hadroproduction. Firstly, it makes the computations faster with a limited loss of generality. Secondly, we have to acknowledge that we do not have at present time a global and consistent theoretical description of inclusive quarkonium production in the whole transverse momentum domain at hadron colliders. Thirdly, most of available models on the market show uncertainties larger

\footnotetext{
3 We stress that a similar procedure could devised for Drell-Yan pair, $W$ and $Z$ production.
}

than those of the data which they are meant to describe (and which sometimes they do not). Some of these observations also apply to $D$ and $B$ production.

This translates into the following advantages:

1. one can describe single quarkonium, $D$ and $B$ production in $p p$ collisions in a very satisfactory way with only $2-3$ tuned parameters for each meson;

2. the uncertainty within our approach is well controlled by the available $p p$ data which, as just said, is much smaller than the theoretical uncertainties of the state-of-the-art calculations;

3. the method is much more efficient to generate events, with significantly reduced Monte Carlo uncertainty, owing to the simplicity of the computation.

\section{1 $p p$ cross section and partonic amplitude}

As for the partonic scattering, we use a functional form for $\overline{\left|\mathcal{A}\left(k_{1} k_{2} \rightarrow \mathcal{H}+k_{3}\right)\right|^{2}}$ initially proposed in [48] and then successfully used in [49-52] to model single quarkonium production at Tevatron and LHC energies in the context of double-parton scattering (DPS) studies. It reads

$$
\begin{aligned}
\overline{\left|\mathcal{A}\left(k_{1} k_{2} \rightarrow \mathcal{H}+k_{3}\right)\right|^{2}}= & \frac{\lambda^{2} \kappa s x_{1} x_{2}}{M_{\mathcal{H}}^{2}} \exp \left(-\kappa \frac{\min \left(P_{T}^{2},\left\langle P_{T}\right\rangle^{2}\right)}{M_{\mathcal{H}}^{2}}\right) \\
& \times\left(1+\theta\left(P_{T}^{2}-\left\langle P_{T}\right\rangle^{2}\right) \frac{\kappa}{n} \frac{P_{T}^{2}-\left\langle P_{T}\right\rangle^{2}}{M_{Q}^{2}}\right)^{-n},
\end{aligned}
$$

where $k_{i}$ denote the partons involved in the hard scattering, $x_{1,2}$ are the momentum fractions carried by $k_{1,2}, s$ is the square of the centre-of-mass energy of the hadron collision, $P_{T}\left(M_{\mathcal{H}}\right)$ is the transverse momentum (mass) of the produced particle, $\mathcal{H}$, and $\theta(x)$ is the Heaviside step function. $\overline{|\mathcal{A}|^{2}}$ is meant to account for the squared amplitude averaged (summed) over the initial (final) helicity/colour factors. It contains 4 parameters $\lambda, \kappa,\left\langle P_{T}\right\rangle, n$, to be determined from the $p p$ experimental data via a fit after the usual convolution with the PDFs:

$$
\begin{aligned}
& \frac{d \sigma(p p \rightarrow \mathcal{H}+X)}{d \Phi_{2}}=\frac{1}{2 s} \int d x_{1} d x_{2} x_{1} \\
& \times f^{p}\left(x_{1}\right) x_{2} f^{p}\left(x_{2}\right) \overline{\left|\mathcal{A}\left(k_{1} k_{2} \rightarrow Q+k_{3}\right)\right|^{2}},
\end{aligned}
$$

where $f^{p}$ denotes the proton PDF and $\Phi_{2}$ is the relativistic two-body phase space measure for the $2 \rightarrow 2$ scattering. The default factorisation scale at which are eveluated the nuclear and proton PDFs is taken as the transverse mass $\left(\sqrt{M_{\mathcal{H}}^{2}+P_{T}^{2}}\right)$ of the particle $\mathcal{H}$ in what follows.

In what follows, we will only consider processes which are dominated by gluon fusion at LHC energies. All the pro- 
Table 1 Results of a fit of $d^{2} \sigma / d P_{T} d y$ of prompt $J / \psi$ in $p p$ collisions using CT10NLO and CT14NLO, where we have fixed the values of $n$ and $\left\langle P_{T}\right\rangle$. (The uncertainties from the $\chi^{2}$ fit below the per cent level are not shown.)

\begin{tabular}{llllll}
\hline PDF & Data & $\lambda$ & $\kappa$ & $\left\langle P_{T}\right\rangle$ & $n$ \\
\hline CT14NLO & LHCb [55,56] & $0.296 \pm 0.118$ & 0.558 & 4.5 (fixed) & 2 (fixed) \\
& ATLAS [57] and CMS [58] & 0.378 & $0.743 \pm 0.0395$ & 4.5 (fixed) & 2 (fixed) \\
CT10NLO & LHCb [55,56] & 0.297 & 0.532 & 4.5 (fixed) & 2 (fixed) \\
& ATLAS [57] and CMS [58] & 0.383 & $0.750 \pm 0.0364$ & 4.5 (fixed) & 2 (fixed) \\
\hline
\end{tabular}

cedure can readily be generalised to other partonic initial states.

\subsection{Accounting for the nuclear PDF impact}

As announced, we will also only consider the nuclear modification of the PDF among the possible effects acting on quarkonia, $D$ and $B$ mesons. Such a restriction would probably not yield a good description of the quarkonium excited states [2], which we therefore do not discuss. Along the same lines, we will focus on the LHC regime where the nuclear absorption is likely negligible. It may not be so at RHIC and even less at fixed-target energies.

Whereas one could think that the proposed procedure can be used to evaluate the sole impact of the nPDF on the excited states or the ground states at lower energies, one may want to be careful that in presence of other significant effects, the impact of the nPDFs may be affected. A clear example is a $b$-dependent anti-shadowing, which would tend to generate more $J / \psi$ in the centre of the overlap zone, which then may have more chance to be broken up by the nuclear absorption than those produced in the periphery of the overlap zone. Yet, the procedure should give a right order of magnitude of the nPDF impact even if other effects are at play.

As it is customary, the yield of a particle $\mathcal{H}$ in $p A$ collisions is obtained from that corresponding to the simple superposition of the equivalent number of $p p$ collisions corrected by a factor encoding the nuclear modification of the parton flux. This is absolutely equivalent to directly using nuclear PDFs (normalised to the nucleus atomic number $A$ ) instead of proton PDFs. As aforementioned, our procedure does not currently rely on a Glauber code and we will thus restrict our studies to minimum bias collisions, i.e. integrated on all possible impact parameters $b$.

As such, the correction factor can be expressed in terms of the ratios $R_{i}^{A}$ of the nuclear PDF (nPDF) in a nucleon belonging to a nucleus $A$ to the PDF in the free nucleon:

$R_{i}^{A}\left(x, Q^{2}\right)=\frac{f_{i}^{A}\left(x, Q^{2}\right)}{A f_{i}^{p}\left(x, Q^{2}\right)}, \quad i=q, \bar{q}, g$

To illustrate the potentiality of our procedure, we will only use two of the most up-to-date nPDF parametrisations resulting from global analyses with uncertainties. The first is EPS09 [27], which provides the fit uncertainties at both leading order (LO, dubbed EPS09LO) and next-to-leading order (NLO, dubbed EPS09NLO) and is available in the library LHAPDF5 [45]. The nPDF effects are given in terms of $R_{i}^{A}\left(x, Q^{2}\right)$ for all the flavours.

A new set, nCTEQ15 [24], has recently been released. It is available in the library LHAPDF6 [47] and provides NLO nuclear PDFs. As such, it is important to use the very same proton PDF as the one used for the fit. We have thus used CT14NLO [53]. In the case of EPS09, which provides ratios, the proton PDF to be used is less critical. In principle, we should have used CTEQ6(L1 or M) by consistency with EPS09, or CT14NLO for a good comparison of the yields with nCTEQ. Since CT14NLO is not available in LHAPDF5 and the code cannot load two PDF libraries at a time, we have preferred to use CT10NLO [54] which anyhow yields very similar gluon PDFs.

\section{Fitting the LHC $p p$ cross sections}

At the LHC, we can essentially divide the inclusive (prompt) $J / \psi$ production cross-sections measurements into 2 classes: the slightly forward and low $P_{T}$ (from 0 up to roughly 20 $\mathrm{GeV}$ ) data of LHCb and ALICE and those from ATLAS and CMS at "high" $P_{T}$ (from 6-8 up to roughly $100 \mathrm{GeV}$ ). ${ }^{4} \mathrm{We}$ have performed 2 times $2 \chi^{2}$ fits of $d^{2} \sigma / d P_{T} d y$ of prompt $J / \psi$ production in $p p$ collisions with 2 PDF sets (CT14NLO and $\mathrm{CT} 10 \mathrm{LO})$ using, on the one hand, LHCb data $[55,56]$ and, on the other, ATLAS [57] and CMS [58] data. The fit parameters $\left(\lambda, \kappa,\left\langle P_{T}\right\rangle\right.$ and $n$ of Eq. 1) are shown in Table 1. A comparison of our fit results with the experimental data is shown in Fig. 1a, d. The procedure is particularly successful,

\footnotetext{
4 ALICE has also measured low $P_{T}$ central $J / \psi$ but with a limited statistical precision and a $b$ feed-down contamination. The forward ALICE data are also prone to such a $b$ feed-down contamination. As such, we will focus on the LHCb data for our fits in the forward and low $P_{T}$ region. We also note that CMS has the capacity to cover $P_{T}$ down to $3 \mathrm{GeV}$ (even below in specific cases) in its most forward/backward acceptance.
} 


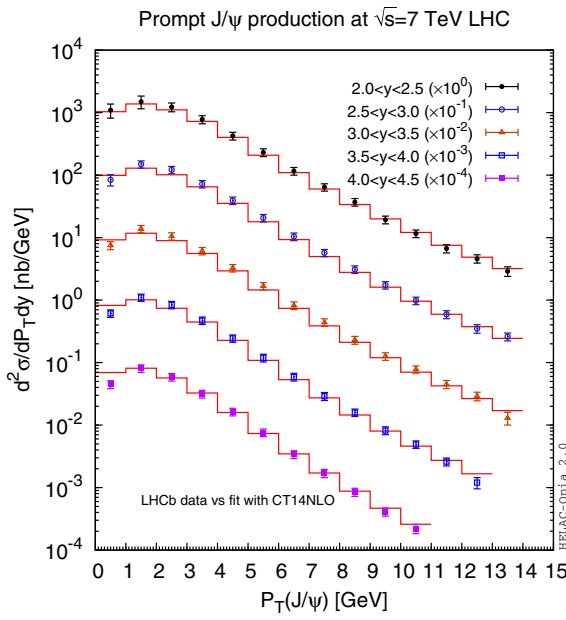

(a) $J / \psi[55]$

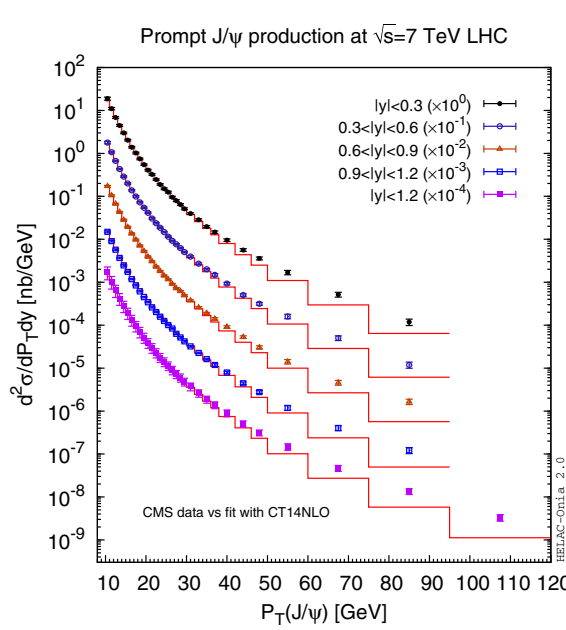

(d) $J / \psi[58]$

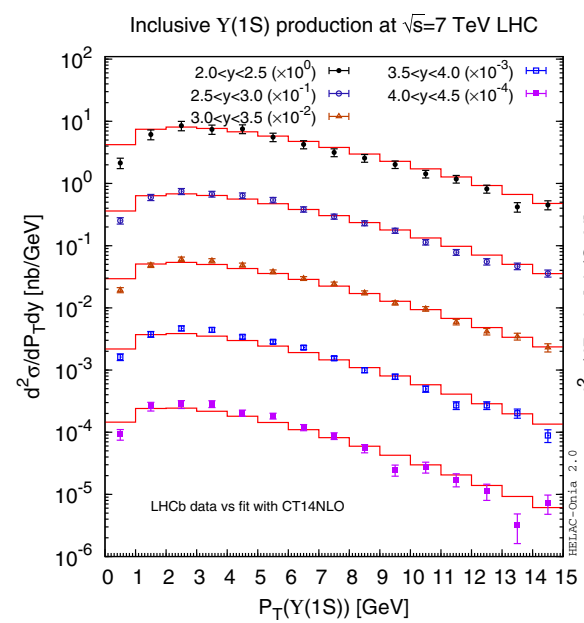

(g) $\Upsilon[61]$

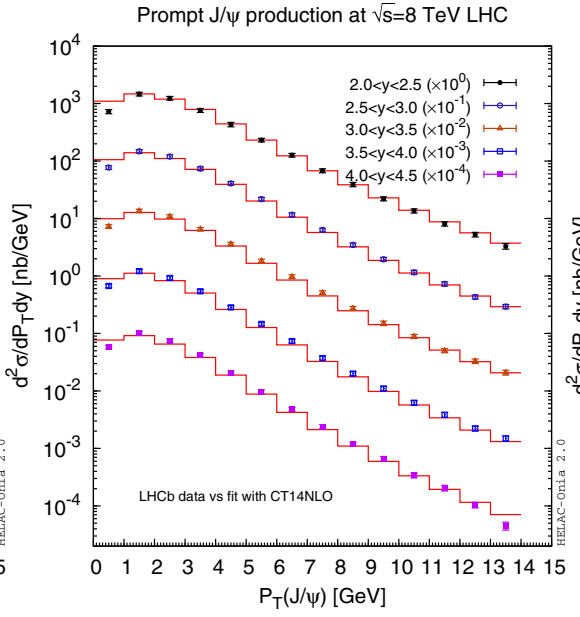

(b) $J / \psi[56]$

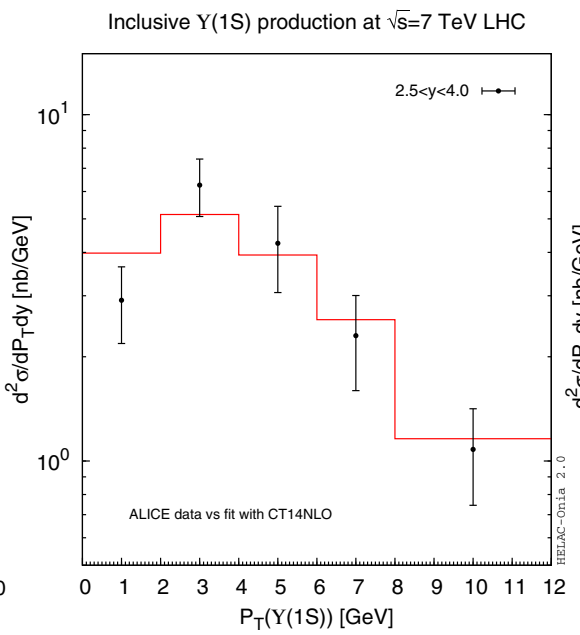

(e) $\Upsilon[59]$

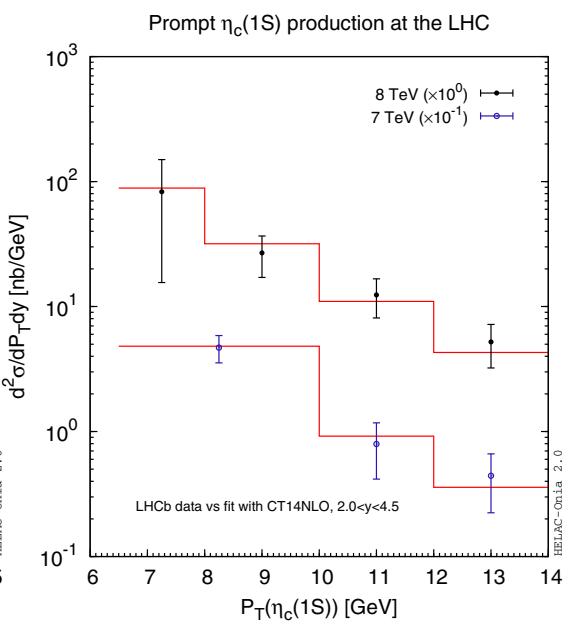

(h) $\eta_{c}[62]$

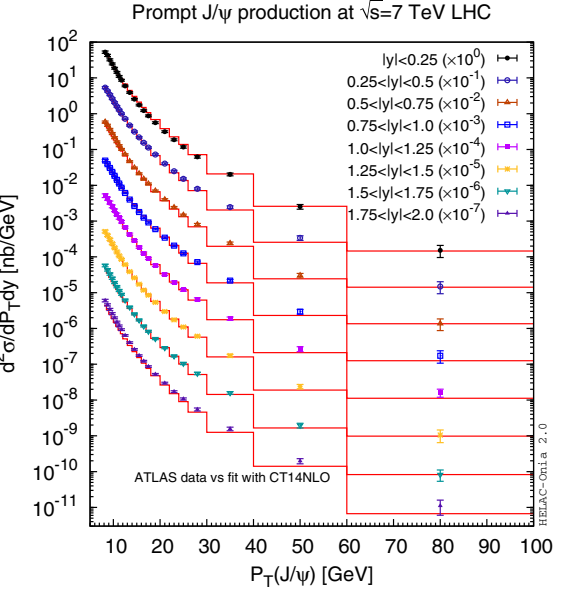

(c) $J / \psi[57]$

Inclusive $\mathrm{Y}(1 \mathrm{~S})$ production at $\sqrt{\mathrm{S}}=7 \mathrm{TeV} \mathrm{LHC}$

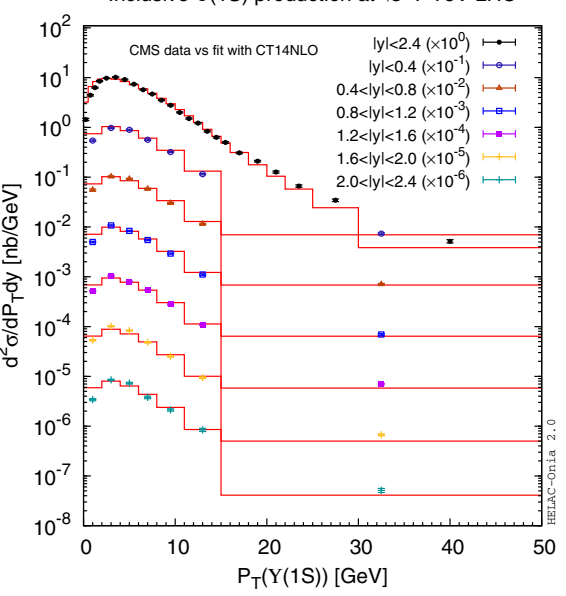

(f) $\Upsilon[60]$

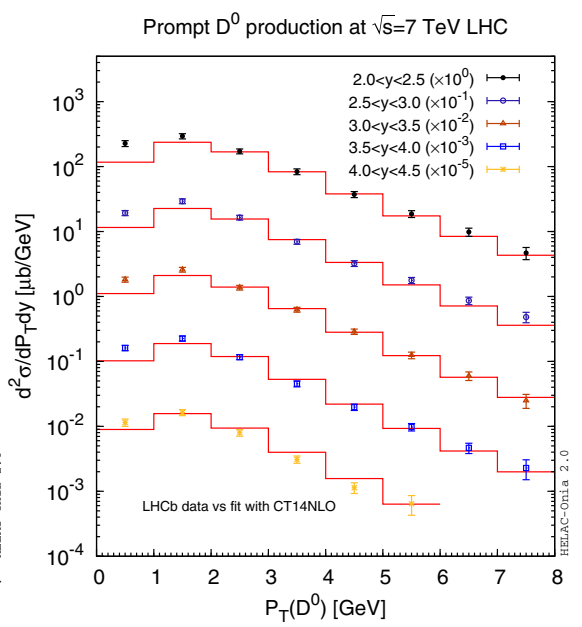

(i) $D^{0}[63]$

Fig. 1 Comparison of our fit results with the prompt $J / \psi(\mathbf{a}-\mathbf{d})$, inclusive $\Upsilon(\mathbf{e}-\mathbf{g})$, prompt $\eta_{c}$ (h) and prompt $D^{0}$ (i) production data in $p p$ collisions at the LHC with CT14NLO as our proton PDF 
Table 2 Results of a fit of $d^{2} \sigma / d P_{T} d y$ of inclusive $\Upsilon(1 S)$ in $p p$ collisions using CT10NLO and CT14NLO, where we have fixed the values of $n$ and $\left\langle P_{T}\right\rangle$. The experimental data used in the fit are from ALICE [59], LHCb [61,64], ATLAS [65] and CMS [60]. (The uncertainties from the $\chi^{2}$ fit below the per cent level are not shown.)

\begin{tabular}{lllll}
\hline PDF & $\lambda$ & $\kappa$ & $\left\langle P_{T}\right\rangle$ & $n$ \\
\hline CT14NLO & 0.768 & $0.0841 \pm 0.0271$ & 13.5 (fixed) & 2 (fixed) \\
CT10NLO & $0.687 \pm 0.367$ & 0.0864 & 13.5 (fixed) & 2 (fixed) \\
\hline
\end{tabular}

Table 3 Results of a fit of $d^{2} \sigma / d P_{T} d y$ of prompt $\eta_{c}(1 S)$ in $p p$ collisions using CT10NLO and CT14NLO, where we have fixed the values of $n$ and $\left\langle P_{T}\right\rangle$. The experimental data used in the fit are from $\mathrm{LHCb}$ [62]. (The uncertainties from the $\chi^{2}$ fit below the per cent level are not shown.)

\begin{tabular}{lllll}
\hline PDF & $\lambda$ & $\kappa$ & $\left\langle P_{T}\right\rangle$ & $n$ \\
\hline CT14NLO & 0.558 & 0.398 & 4.5 (fixed) & 2 (fixed) \\
CT10NLO & 0.337 & 0.291 & 4.5 (fixed) & 2 (fixed) \\
\hline
\end{tabular}

Table 4 Results of a fit of $d^{2} \sigma / d P_{T} d y$ of prompt $D^{0}$ in $p p$ collisions using CT10NLO and CT14NLO, where the value of $n$ was fixed. The experimental data used in the fit are from LHCb [63]. (The uncertainties from the $\chi^{2}$ fit below the per cent level are not shown.)

\begin{tabular}{lllll}
\hline PDF & $\lambda$ & $\kappa$ & $\left\langle P_{T}\right\rangle$ & $n$ \\
\hline CT14NLO & 2.29 & 1.11 & 0.88 & 2 (fixed) \\
CT10NLO & 2.38 & 1.62 & 0.521 & 2 (fixed) \\
\hline
\end{tabular}

but for a few marginal bins. ${ }^{5}$ These will nevertheless do not have a visible impact on the $p A$ observables to be discussed later.

For the $\Upsilon(1 S)$ case, all the experiments have access to low $P_{T}$ data and there is no $b$ feed-down contamination. We have performed 2 fits (with CT14NLO and CT10LO) using data from ALICE [59], LHCb [61,64], ATLAS [65] and CMS [60] altogether. See Table 2 for the fit results and Fig. 1e-h for comparison with the fit spectra.

For the prompt $\eta_{c}$ case, we have performed 2 fits (with CT14NLO and CT10LO) from the sole LHCb [62] data. See Table 3 for the fit results and Fig. $1 \mathrm{~h}$ for comparison with the fit spectra.

As for the $D^{0}$, we have performed 2 fits (with CT14NLO and CT10LO) from the LHCb [63] data. See Table 4 for the fit results and Fig. 1i for comparison with the fit spectra.

Just as for the $J / \psi$ fits, the procedure works very well for $\Upsilon(1 S), \eta_{c}$ and $D^{0}$ and gives us confidence that using the corresponding parametrised squared amplitudes will provide us with a reliable mapping of the $x_{1,2}, y$ and $P_{T}$ space.

\footnotetext{
5 Let us in particular note the slight discrepancy with the CMS very high $P_{T}$ data. The very same fits are however consistent with the ATLAS data in the same $P_{T}$ regime, which possibly indicates an underestimation of the systematical experimental uncertainties in that regime.
}
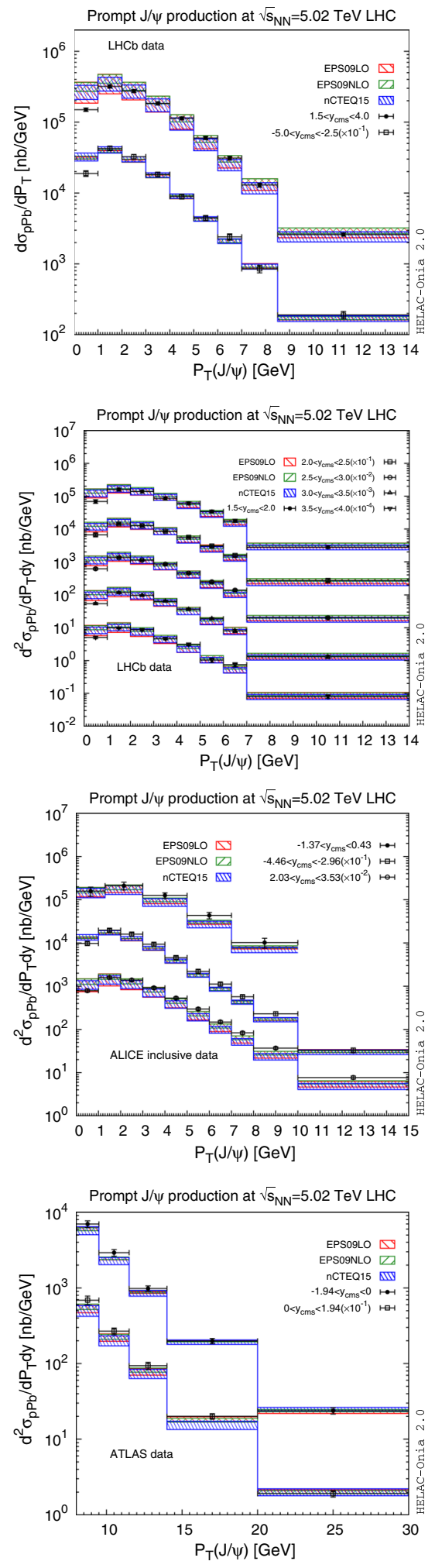

Fig. $2 d \sigma / d P_{T}$ for prompt $J / \psi$ production in $p \mathrm{~Pb}$ collisions at $\sqrt{s_{N N}}=5.02 \mathrm{TeV}$ : comparison between our results and the measurements of LHCb [66], ALICE [67] and ATLAS [68] 

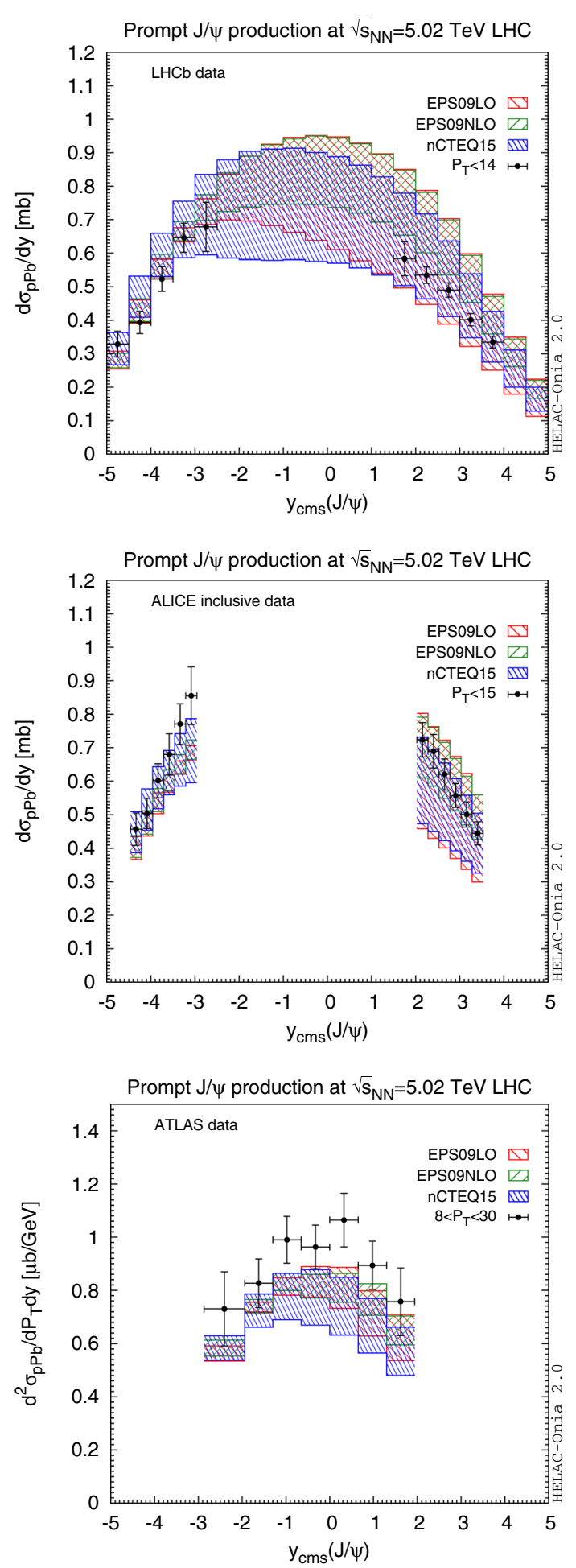

Fig. 3 Rapidity dependence of the cross-section for prompt $J / \psi$ production in $p \mathrm{~Pb}$ collisions at $\sqrt{s_{N N}}=5.02 \mathrm{TeV}$ : comparison between our results and the measurements of LHCb [66], ALICE [69] and ATLAS [68]

\section{Results}

4.1 Rapidity and transverse-momentum dependence of the production cross-section in $p \mathrm{~Pb}$ collisions at $\sqrt{s_{N N}}=5.02 \mathrm{TeV}$

Now that we have described our approach, we can present our results for the cross-section for quarkonium and $D^{0}$ production in proton-lead $(p \mathrm{~Pb})$ collisions at the LHC. In the following, we show comparisons with all the existing data. Our histograms are calculated under the same cuts as the experimental data. As announced, we have employed three different nPDF EPS09LO, EPS09NLO and nCTEQ15. In all the following plots, the uncertainty bands represent the nuclear PDF uncertainty only. In particular, we have not varied the factorisation scale despite the fact that it can indeed alter our results (see Sect. 4.4 for a short discussion).

The transverse-momentum $P_{T}$ spectra $\left(d \sigma_{p \mathrm{~Pb}} / d P_{T}\right)$ of promptly produced $J / \psi$ in $p \mathrm{~Pb}$ collisions at $\sqrt{s_{N N}}=5.02$ $\mathrm{TeV}$ are shown in Fig. 2. Comparisons are made with the LHCb prompt $J / \psi$ production data [66] in both the forward $\left(1.5<y_{\text {c.m.s. }}^{J / \psi}<4.0\right)^{6}$ and backward $(-5.0<$ $\left.y_{\text {c.m.s. }}^{J / \psi}<-2.5\right)$ rapidity regions in Fig. $2 \mathrm{a}$. Figure $2 \mathrm{~b}$ shows a comparison with the double differential cross sections $d^{2} \sigma_{p \mathrm{~Pb}} / d P_{T} d y$ of $J / \psi$ production of LHCb. Similarly, comparisons with the ALICE data [67] and ATLAS data [68] are given in Fig. 2c, d respectively. We note that ALICE data do not exclude the contribution from $b$-hadron decays. In general, the agreement with the yields differential in $P_{T}^{J / \psi}$ is satisfactory both at low $P_{T}$ and high $P_{T}$.

In Fig. 3, we have compared the LHCb [66], ALICE [69] and ATLAS data [68] with the $J / \psi$ cross-section differential in $y$. It is interesting to notice that the results with the three nPDF show different uncertainties. In the forward region (low $x_{2}$ ), the result with EPS09NLO has the smallest uncertainty and tend to overshoot the LHCb data [66] (see Fig. 3a). Such a discrepancy does not appear in Fig. 3b. One can also note that the EPS09LO uncertainty can be considered as the combination of both EPS09NLO and nCTEQ15 uncertainties in the forward region. In the backward region, owing to both the significant experimental and nPDF uncertainties, the three nPDFs are compatible with the data. At high $P_{T}$ (in Fig. 3c for the ATLAS data [68]), although the central values of the experimental data are systematically higher than our theoretical bands, they remain compatible within one standard deviation. There could indeed be an overestimation of the nPDF suppression in this region or an offset in the ATLAS data.

\footnotetext{
6 Unless indicated, all rapidity $y$ (or $y_{\text {c.m.s. }}$ ) mean the rapidity in the centre-of-mass frame of nucleon-nucleon collision. In particular, rapidities in the laboratory frame would read $y_{\text {lab. }}$.
} 


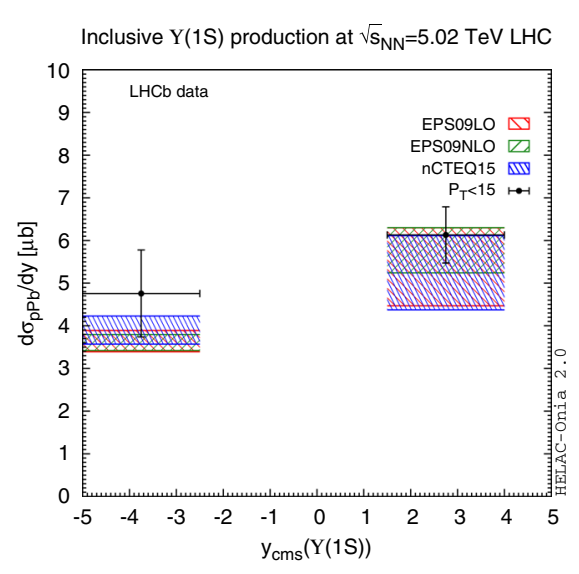

(a)
Inclusive $\mathrm{Y}(1 \mathrm{~S})$ production at $\sqrt{\mathrm{S}}_{\mathrm{NN}}=5.02 \mathrm{TeV} \mathrm{LHC}$

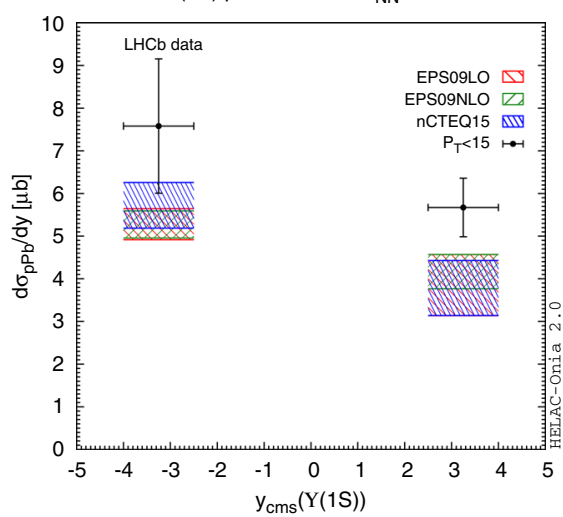

(b)
Inclusive $\mathrm{Y}(1 \mathrm{~S})$ production at $\sqrt{\mathrm{S}}_{\mathrm{NN}}=5.02 \mathrm{TeV} \mathrm{LHC}$

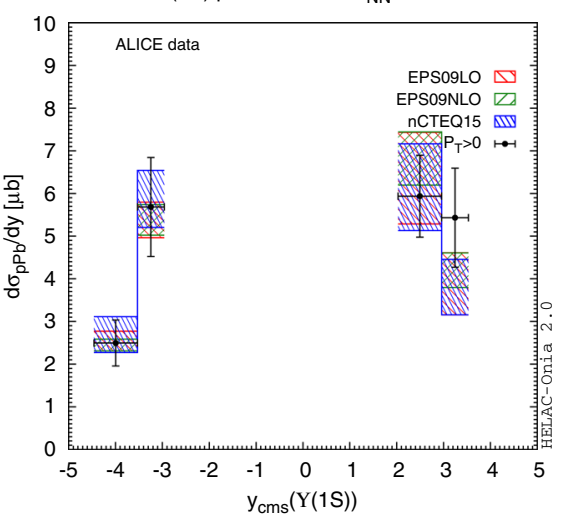

(c)

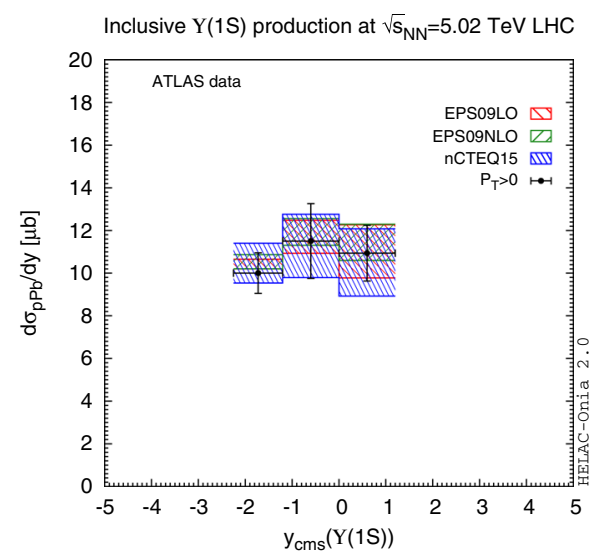

(d)

Fig. 4 Differential cross-section for inclusive $\Upsilon(1 S)$ production in $p \mathrm{~Pb}$ collisions at $\sqrt{s_{N N}}=5.02 \mathrm{TeV}$ : comparison of a-d the rapidity dependence obtained with our procedure with the measurements

As for the $\Upsilon(1 S)$, Fig. 4a, b show comparisons with the $\mathrm{LHCb}$ data. The agreement is better when the full $\mathrm{LHCb}$ range is considered as opposed to that when the LHCb acceptance is restricted to a range where equal positive and negative $y$ can be accessed (Fig. 4b). A good agreement is also obtained with the ALICE data (Fig. 4c) in a similar rapidity domain. In the ATLAS acceptance, all three nPDF magnitudes correctly account for the yield differential in $y$ and $P_{T}$ (Fig. 4d, e).

Figures 5 and 6 show comparisons for the $D^{0}$ case between our results for the $3 \mathrm{nPDF}$ and the LHCb and ALICE measurements. The agreement is overall good. The yields tend to lie on the upper half of the uncertainty band. The nPDF uncertainties are however larger than for the quarkonia owing to the smaller value of the factorisation scale. As for the discrepancy in the first $P_{T}$ bin of Fig. 5, one should be careful that our $p p$ parametrisation is not optimal to describe it as well (see Fig. 1i) and tend to undershoot the $p p$ yield.

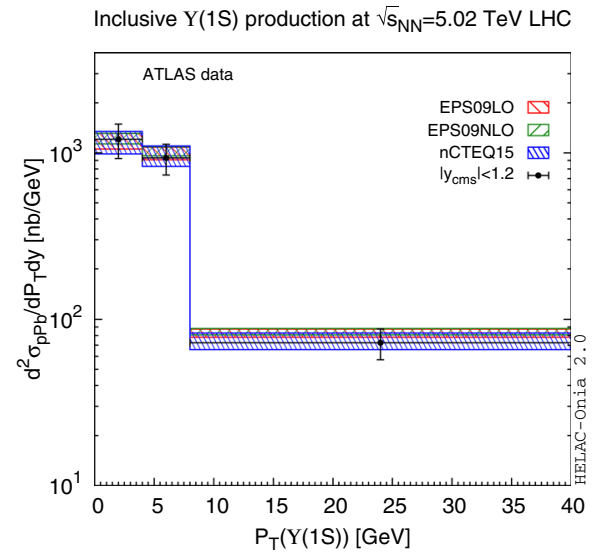

(e)

by LHCb [70], ALICE [71] and ATLAS [72] and e the transversemomentum dependence as measured by ATLAS [72]

Finally, Fig. 7 show predictions - the first ever in the literature - for the $P_{T}$ and $y$ differential yield of $\eta_{c}$ in the $\mathrm{LHCb}$ acceptance.

\subsection{Rapidity and transverse-momentum dependence of $R_{p \mathrm{~Pb}}$ at $\sqrt{s_{N N}}=5.02 \mathrm{TeV}$}

We now present and discuss our results for the nuclear modification factor $R_{p \mathrm{~Pb}}$ which characterises the yield modification of a given probe, say $\mathcal{H}$, in $p \mathrm{~Pb}$ collisions relative to $p p$ collisions. It is the ratio obtained by normalising the $\mathcal{H}$ yield in $p \mathrm{~Pb}$ collisions to the $\mathcal{H}$ yield in $p p$ collisions in the same kinematical conditions ( $y, P_{T}$, nucleon-nucleon energy, etc.) times the average number of binary inelastic nucleon-nucleon collisions. When minimum bias collisions are considered, that is when all the possible geometrical configurations are summed over, it simplifies to the ratio of cross sections corrected by the atomic number of the nucleus $(A=208$ for $\mathrm{Pb})$ : 

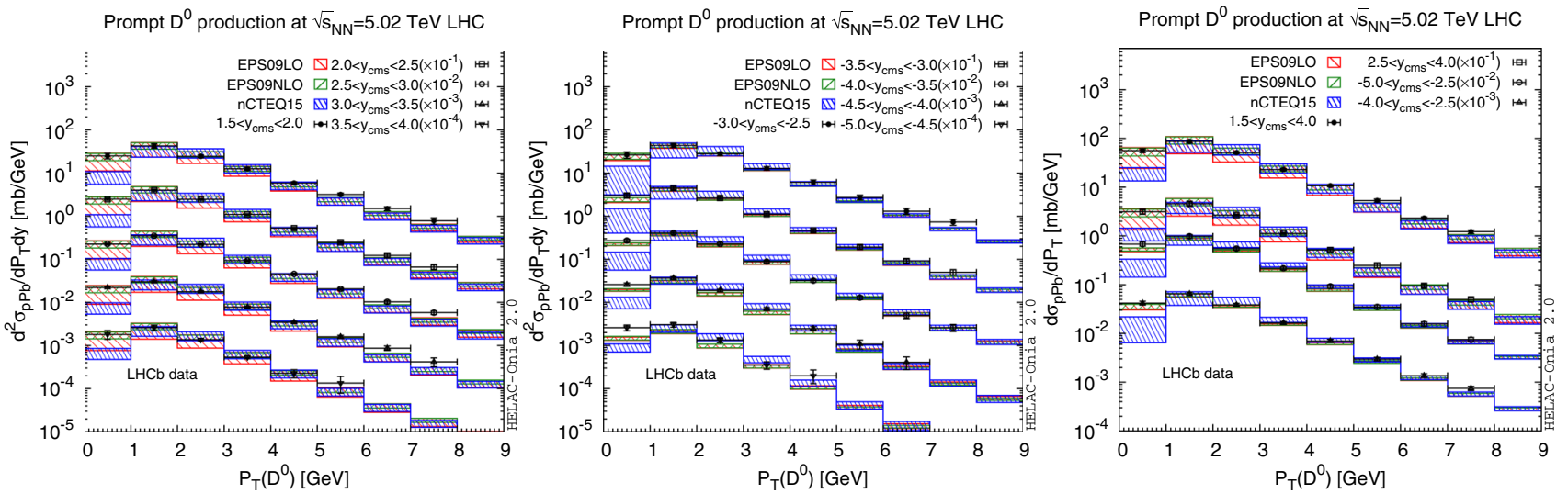

Fig. 5 Transverse-momentum dependence of the production cross-section of promptly produced $D^{0}$ in $p \mathrm{~Pb}$ collisions at $\sqrt{s_{N N}}=5.02$ TeV: comparison between our results and the measurements by $\mathrm{LHCb}[73]$

Fig. 6 a Rapidity (b

Transverse-momentum) dependence of the cross-section for promptly produced $D^{0}$ in $p \mathrm{~Pb}$ collisions at $\sqrt{s_{N N}}=5.02$ $\mathrm{TeV}$ : comparison between our results and the measurements by LHCb [73] (b ALICE [74])

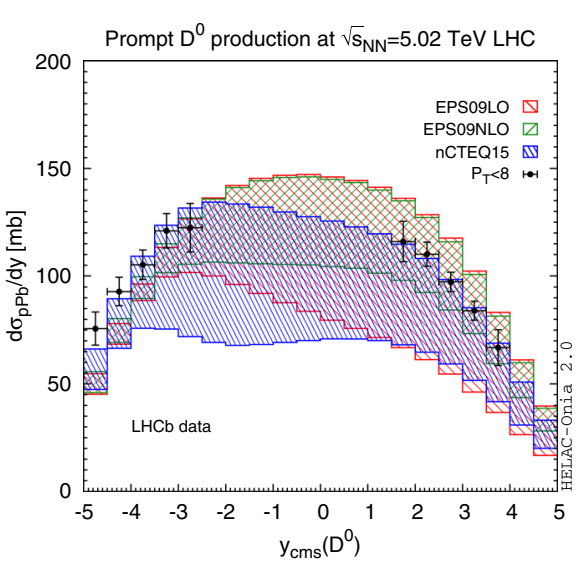

(a) LHCb [73]

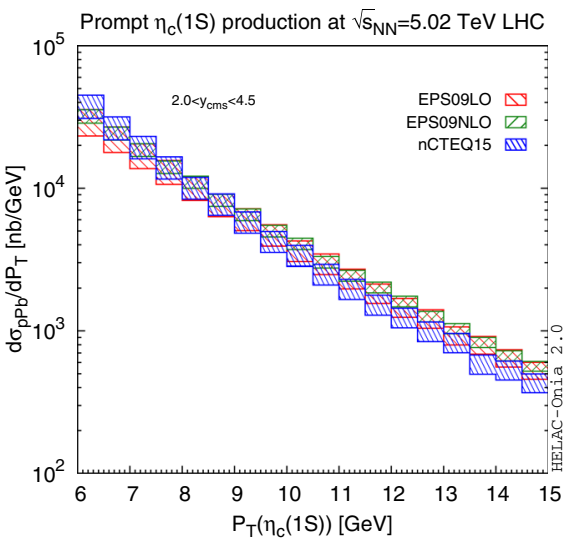

(a)

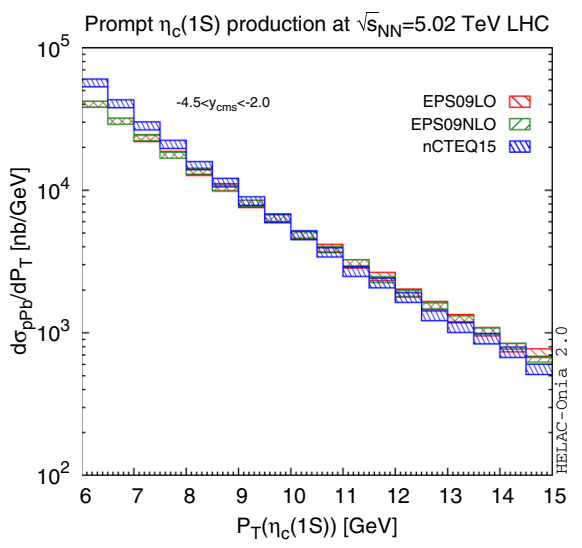

(b)

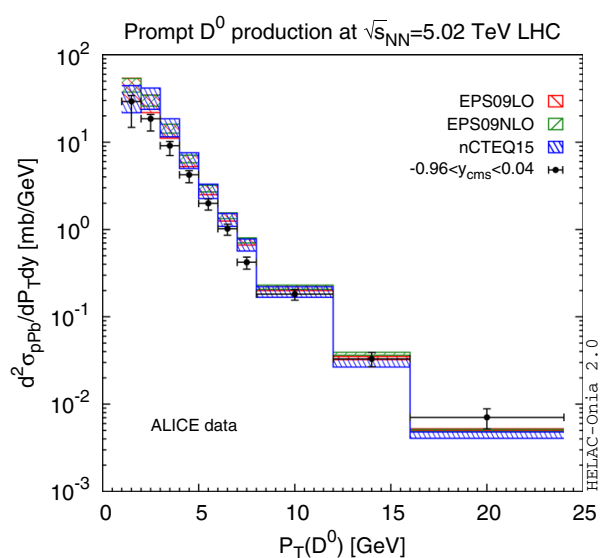

(b) ALICE [74]

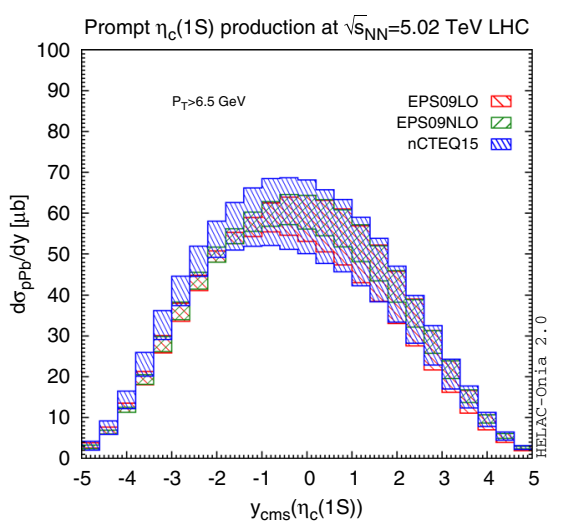

(c)

Fig. 7 a, b Transverse-momentum (c rapidity) dependence of the production cross-section of prompt $\eta_{c}(1 S)$ in $p \mathrm{~Pb}$ collisions at $\sqrt{s_{N N}}=5.02$ $\mathrm{TeV}$. (The uncertainty bands represent the nuclear PDF uncertainty only.)

$R_{p \mathrm{~Pb}}=\frac{d \sigma_{p \mathrm{~Pb}}^{\mathcal{H}}}{A d \sigma_{p p}^{\mathcal{H}}}$.

We first discuss the rapidity dependence of $R_{p \mathrm{~Pb}}$ at the LHC with $\sqrt{s_{N N}}=5.02 \mathrm{TeV}$ for $J / \psi$ production. Our results obtained for the three nPDFs, EPS09LO, EPS09NLO and nCTEQ15 with their associated uncertainties are compared in Fig. 8 to the different experiments. Figure $8 \mathrm{a}, \mathrm{b}$ show low $P_{T}$ data $[66,67,69]$. It is expected that the suppression in the forward region is due to the shadowing effect, 


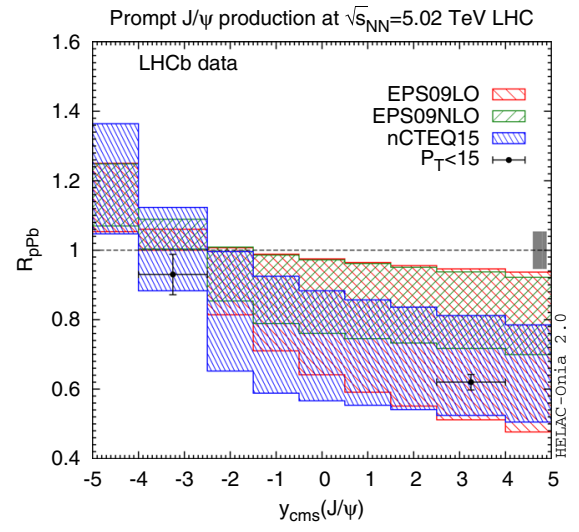

(a)

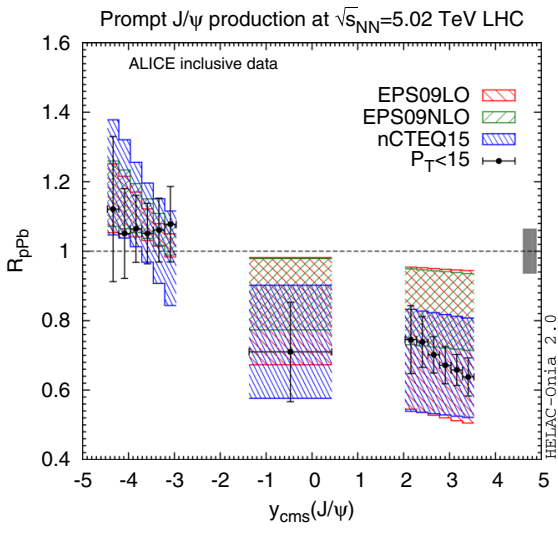

(b)

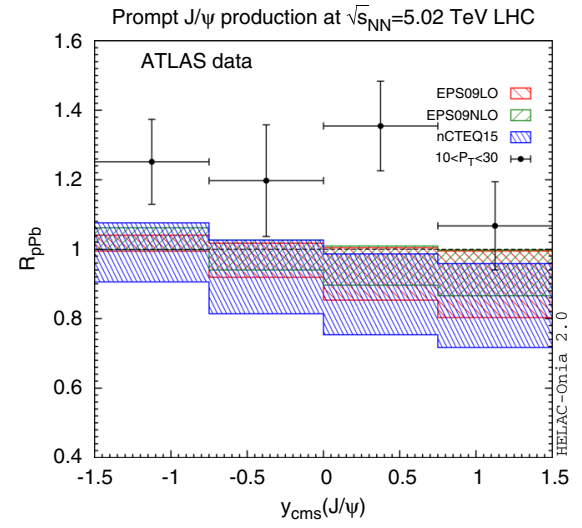

(c)

Fig. 8 Rapidity dependence of $R_{p} \mathrm{~Pb}$ of prompt $J / \psi$ in $p \mathrm{~Pb}$ collisions at $\sqrt{s_{N N}}=5.02 \mathrm{TeV}$ : comparison between our results and the measurements by LHCb [66], ALICE [67,69] and ATLAS [75]

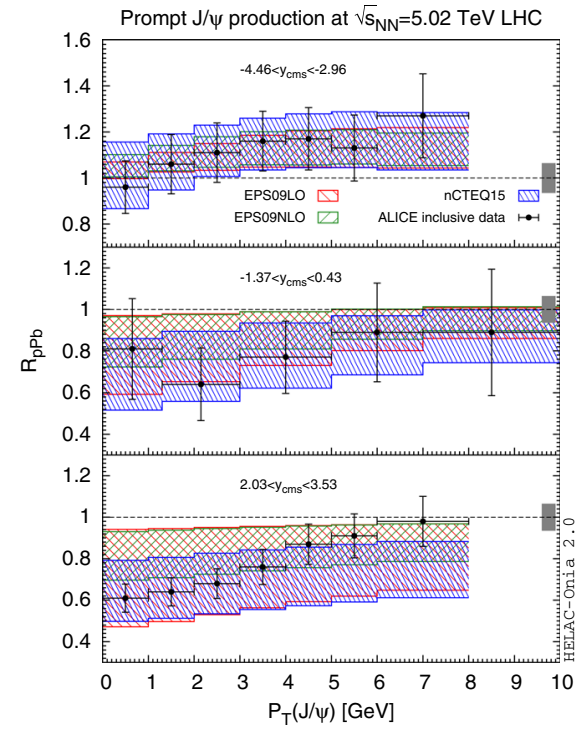

(a)

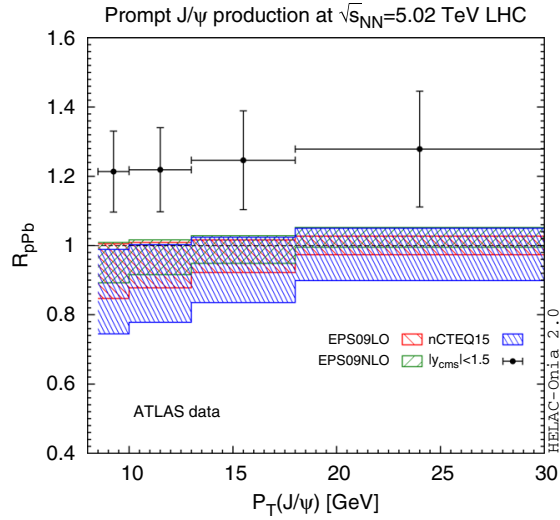

(b)

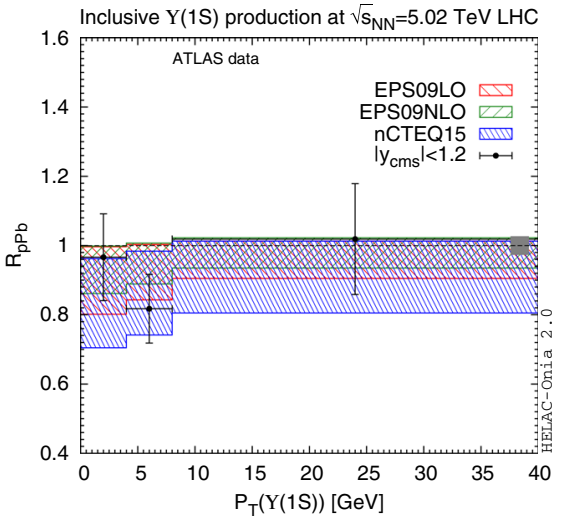

(c)

Fig. 9 a, b $P_{T}$ dependence of $R_{p \mathrm{~Pb}}$ of prompt $J / \psi$ [c inclusive $\left.\Upsilon(1 S)\right]$ in $p \mathrm{~Pb}$ collisions at $\sqrt{s_{N N}}=5.02$ TeV : comparison between our results and the measurements by ALICE [67] and ATLAS [75] (c ATLAS [72])

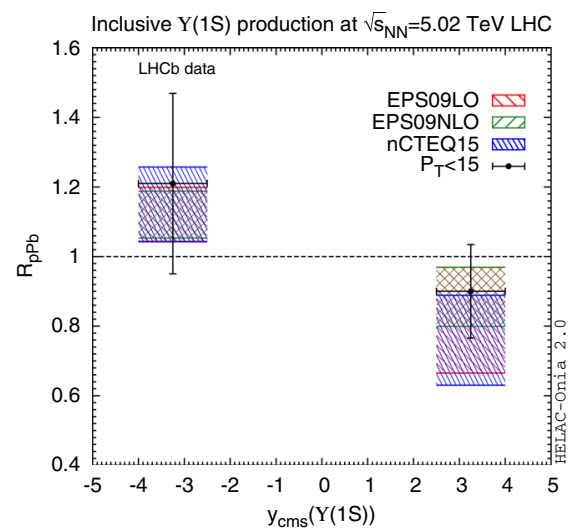

(a)

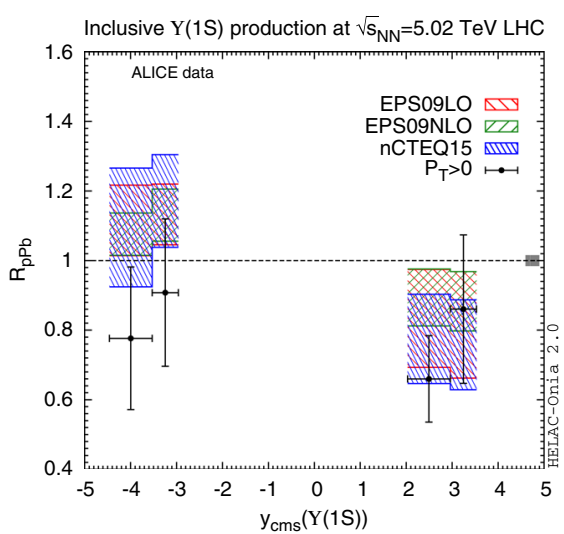

(b)

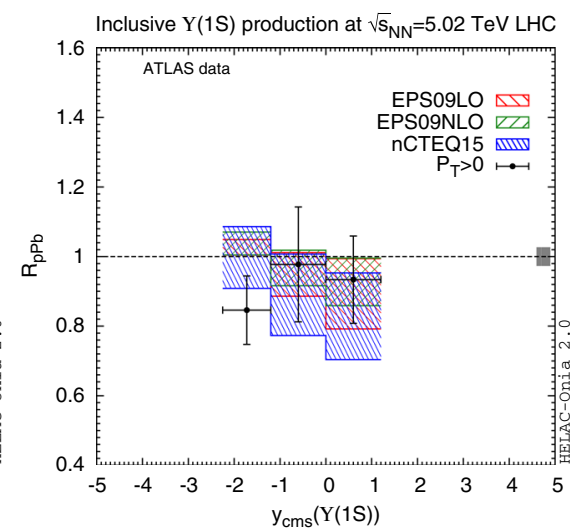

(c)

Fig. 10 Rapidity dependence of $R_{p \mathrm{~Pb}}$ of inclusive $\Upsilon(1 S)$ in $p \mathrm{~Pb}$ collisions at $\sqrt{s_{N N}}=5.02 \mathrm{TeV}$ : comparison between our results and the measurements by LHCb [70], ALICE [71] and ATLAS [72] 


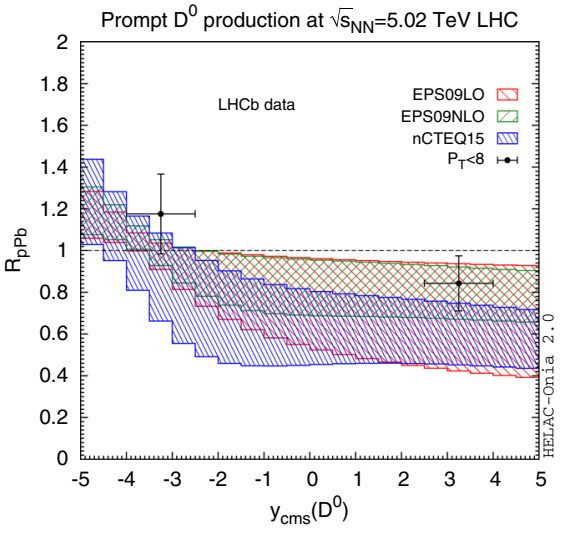

(a)

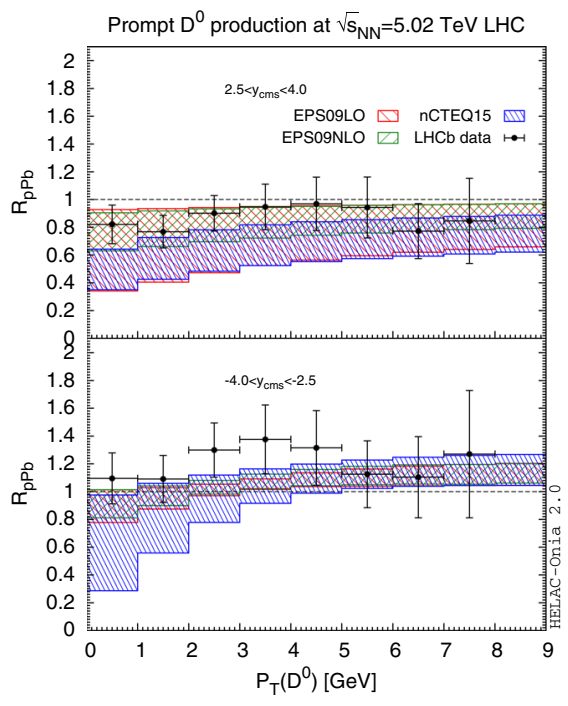

(b)

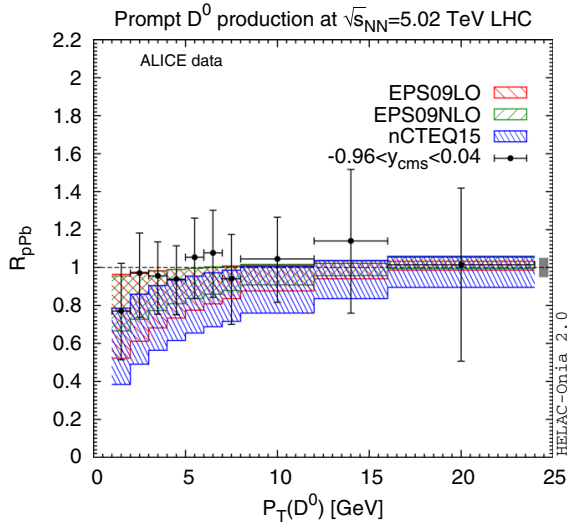

(c)

Fig. 11 Rapidity (a) and transverse-momentum (b, c) dependence of $R_{p \mathrm{~Pb}}$ of promptly produced $D^{0}$ in $p \mathrm{~Pb}$ collisions at $\sqrt{s_{N N}}=5.02 \mathrm{TeV}$ : comparison between our results and the measurements by LHCb [73] (a, b) and ALICE [74] (c)

while the enhancement in the backward region is due to the anti-shadowing effect. The experimental data are compatible with these expectations. Among the three different nPDFs, the data tend to favour the result obtained with nCTEQ15.

It is also interesting to note that the precision of the current data is already better than the nPDF uncertainties, especially in the forward region. This gives some hope that these measurements could ultimately be used to constrain the gluon density in heavy ions, provided that the impact of other nuclear effects could be disentangled. We also note that the shaded boxes on the right of the first two plots refer to the global systematical uncertainty. Such an information is not available for the ATLAS data. A good agreement with the LHCb and ALICE data is obtained; a slight discrepancy with the ATLAS data is observed. It is not clear whether it could be attributed to an offset in the data normalisation. In Fig. 9, we show further comparisons of $R_{p \mathrm{~Pb}}$ vs $P_{T}^{J / \psi}$ between our curves and the ALICE [67] and ATLAS [75] data. Similar to the rapidity distribution, a slight discrepancy is observed in Fig. $9 b$.

Similar comparisons are shown for $\Upsilon(1 S)$ on Figs. $9 \mathrm{c}$ and 10. The overall agreement is acceptable given the large nPDF and experimental uncertainties. Further comparisons with the $D^{0}$ results are presented on Fig. 11 . The agreement is also satisfactory and seems to indicate that EPS09 NLO is providing the best predictions. We however postpone further conclusions to the discussion of the $R_{\mathrm{FB}}$ results which however do not necessarily confirm this observation. To complete this exhaustive list of comparisons, we present our predictions for $R_{p \mathrm{~Pb}}$ of $\eta_{c}$ in the LHCb acceptance of its $p p$ analysis on Fig. 12. We are hopeful that it will motivate the first ever experimental studies of $\eta_{c}$ in $p \mathrm{~Pb}$ collisions at the LHC.
4.3 Rapidity and transverse-momentum dependence of $R_{\mathrm{FB}}$ at $\sqrt{s_{N N}}=5.02 \mathrm{TeV}$

In this section, we discuss the forward-to-backward production ratio $R_{\mathrm{FB}}$ which results from the asymmetry of the proton-nucleus collision and is thus also sensitive to the nuclear effects. In addition, it has the advantage to be a ratio in which many of the systematic uncertainties of the data cancel, in particular that from the $p p$ yield or cross section. It is defined as

$R_{\mathrm{FB}}=\frac{R_{p \text { Pb }}\left(y_{\text {c.m.s. }}>0\right)}{R_{p \text { Pb }}\left(y_{\text {c.m.s. }}<0\right)}=\frac{d \sigma_{p \mathrm{~Pb}}^{\mathcal{H}}\left(y_{\text {c.m.s. }}>0\right)}{d \sigma_{p \text { Pb }}^{\mathcal{H}}\left(y_{\text {c.m.s. }}<0\right)}$,

where the "forward" direction was defined as the flight direction of the proton beam.

We stress that $R_{\mathrm{FB}}$ is identically unity at $y_{\mathrm{c} . \mathrm{m} . \mathrm{s} .}=0$. It tends to remain close to one if the nuclear effects cancel between the forward and backward regions, otherwise, it tends to increase more or less quickly for increasing $\left|y_{\text {c.m.s. }}\right|$. We further note that in the current implementation of our code the nPDF uncertainties in $R_{\mathrm{FB}}$ are generally smaller than in $R_{p} \mathrm{~Pb}$ (or in the cross sections). Indeed, our current code uses the same nPDF eigenset to compute the forward and the backward yields used in a given ratio. This amounts to consider that the uncertainties in the $R_{p \mathrm{~Pb}}$ are correlated. This interpretation (or rather use) of the information given by the nPDF is not unique and we could have considered that the $\mathrm{nPDF}$ uncertainties in $R_{p \mathrm{~Pb}}$ in the forward and backward regions are not necessarily correlated (as the widespread use of theory "bands" may suggest). Doing so, the uncertainties in $R_{\mathrm{FB}}$ would have been significantly larger. Finally, we recall 


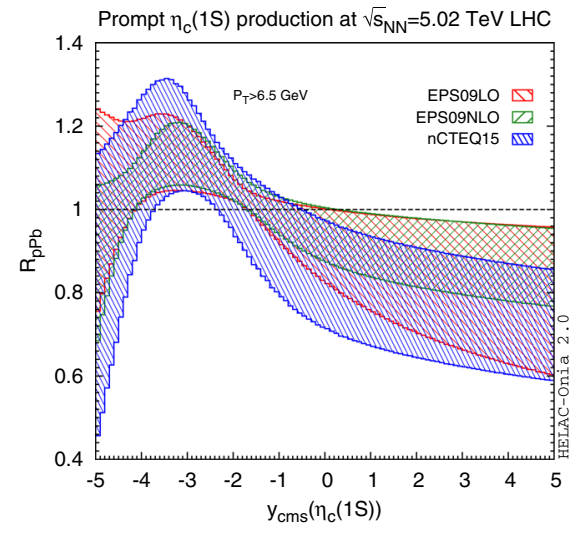

(a)

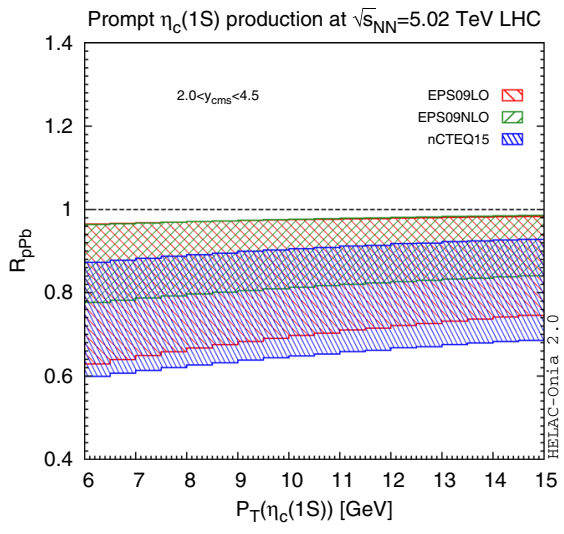

(b)

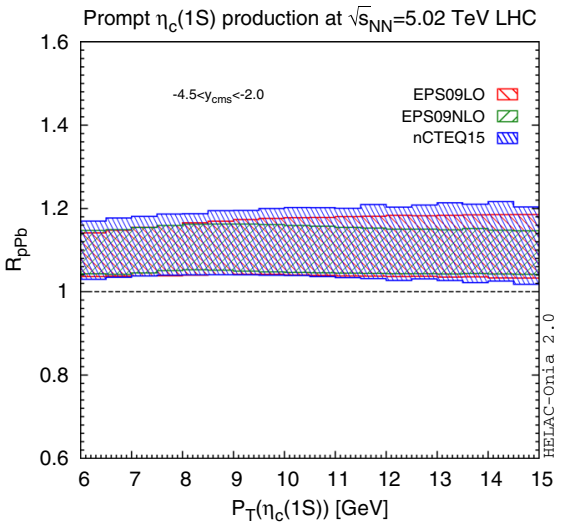

(c)

Fig. 12 Rapidity (a) and transverse-momentum (b, c) dependence of $R_{p \mathrm{~Pb}}$ of prompt $\eta_{c}(1 S)$ in $p \mathrm{~Pb}$ collisions at $\sqrt{s_{N N}}=5.02 \mathrm{TeV}$

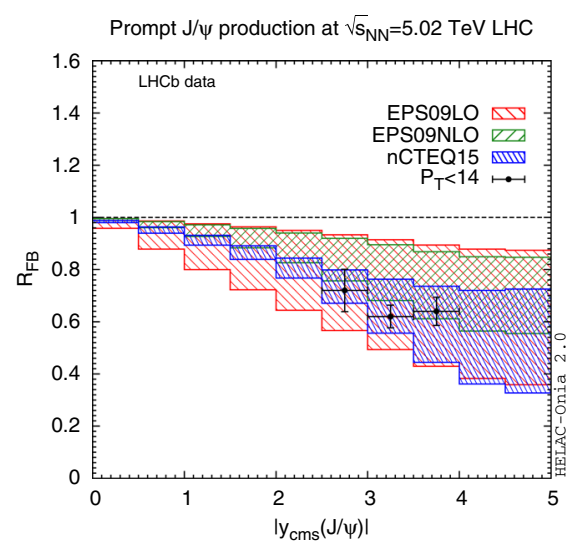

(a) $\mathrm{LHCb}[66]$

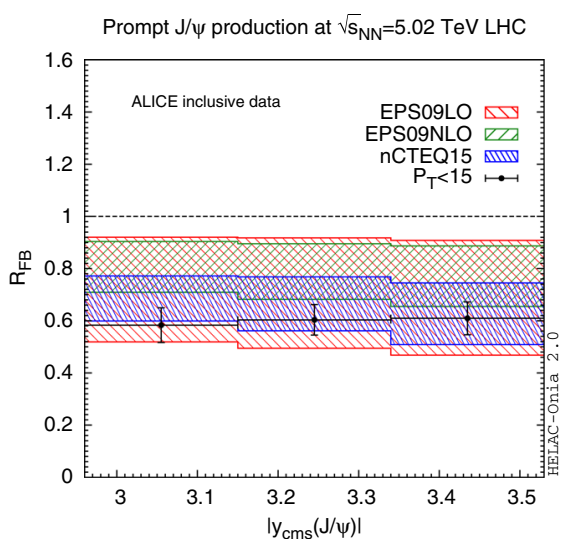

(b) ALICE [69]

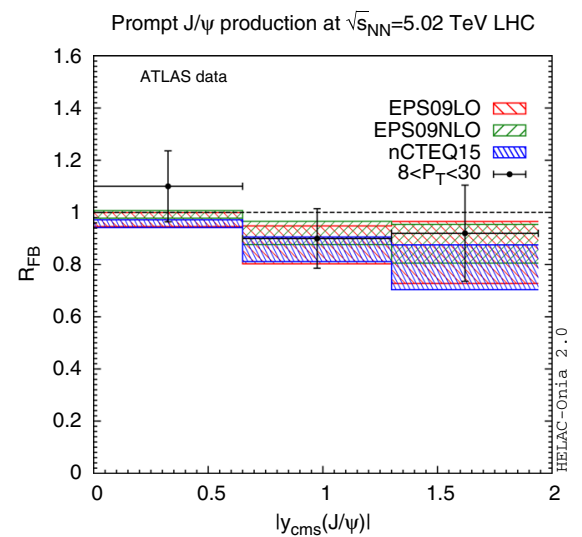

(c) ATLAS [68]

Fig. 13 Rapidity dependence of $R_{\mathrm{FB}}$ of $J / \psi$ in $p \mathrm{~Pb}$ collisions at $\sqrt{s_{N N}}=5.02 \mathrm{TeV}$ : comparison between our results and the measurements by LHCb [66], ALICE [69] and ATLAS [68]. (The uncertainty bands represent the nuclear PDF uncertainty only. ALICE data are for inclusive $J / \psi$.)

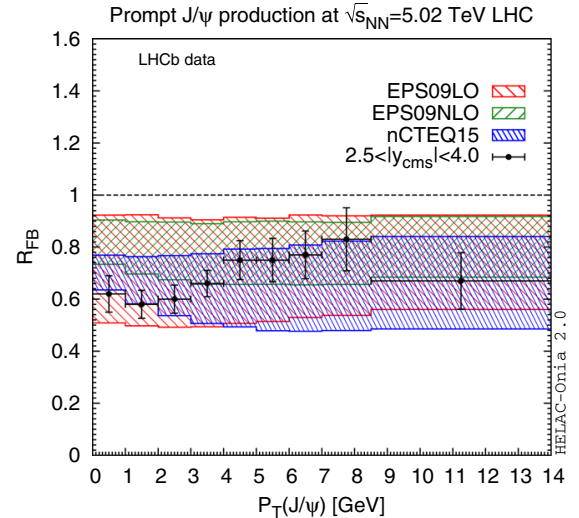

(a)

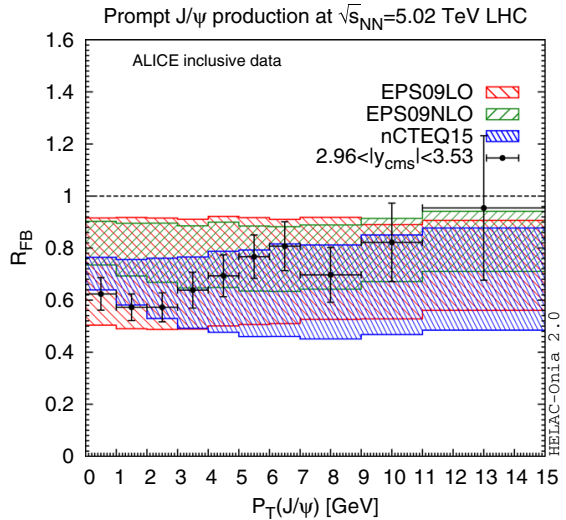

(b)

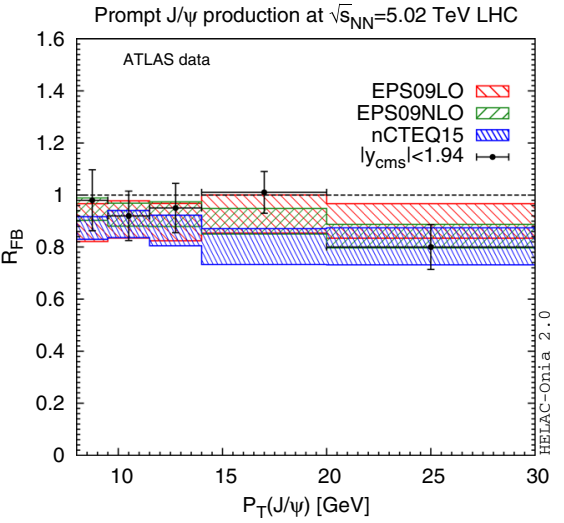

(c)

Fig. 14 Transverse-momentum dependence of the $R_{\mathrm{FB}}$ of $J / \psi$ in $p \mathrm{~Pb}$ collisions at $\sqrt{s_{N N}}=5.02 \mathrm{TeV}$ : comparison between our results and the LHCb [66], ALICE [69] and ATLAS [68] data

that the global systematical uncertainties in the experimental data do cancel. On the experimental side, these results are usually much more reliable.
Figure 13 displays our results for the rapidity dependence of $R_{\mathrm{FB}}$ for the three gluon nPDFs used before (EPS09LO, EPS09NLO, nCTEQ15). For the low $P_{T}$ data of LHCb and 
Fig. 15 Rapidity dependence of $R_{\mathrm{FB}}$ of inclusive $\Upsilon(1 S)$ in $p \mathrm{~Pb}$ collisions at $\sqrt{s_{N N}}=5.02$ $\mathrm{TeV}$ : comparison between our results and the measurements of LHCb [70] and ALICE [71]
Fig. 16 Rapidity (a) and transverse-momentum (b) dependence of $R_{\mathrm{FB}}$ of prompt $D^{0}$ production in $p \mathrm{~Pb}$ collisions at $\sqrt{s_{N N}}=5.02 \mathrm{TeV}$ : comparison between our results and the measurements by $\mathrm{LHCb}[73]$

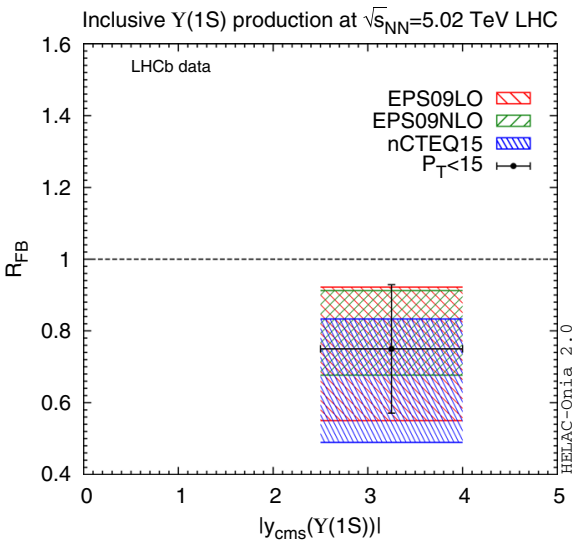

(a)

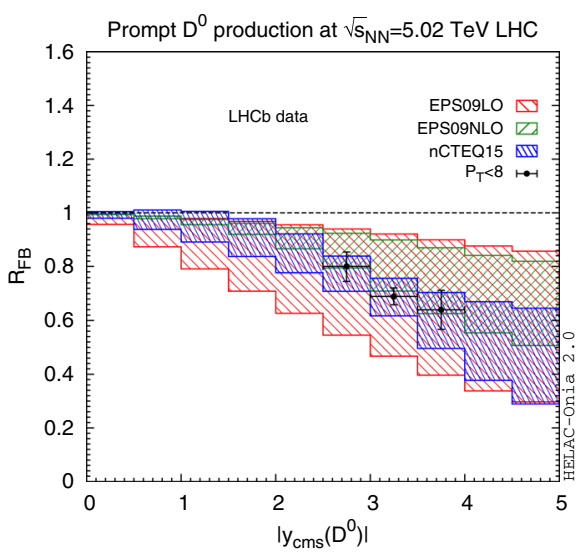

(a)

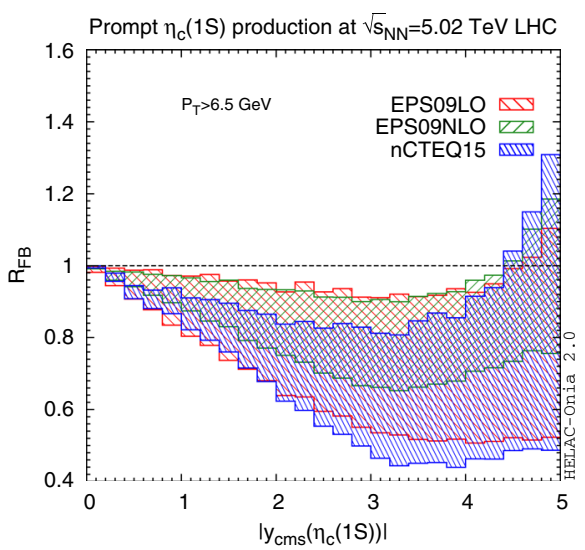

(a)

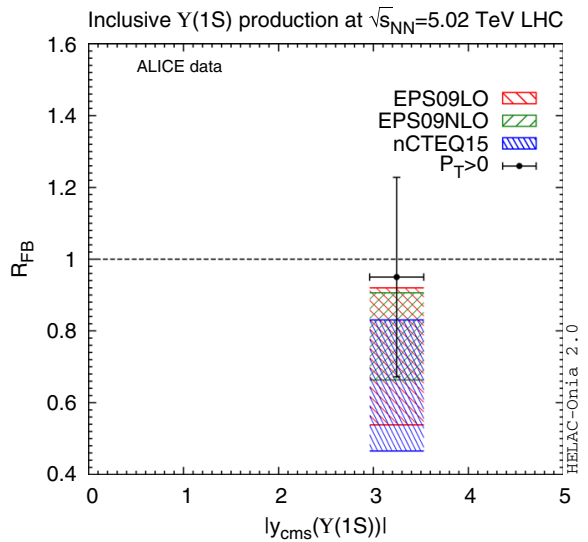

(b)

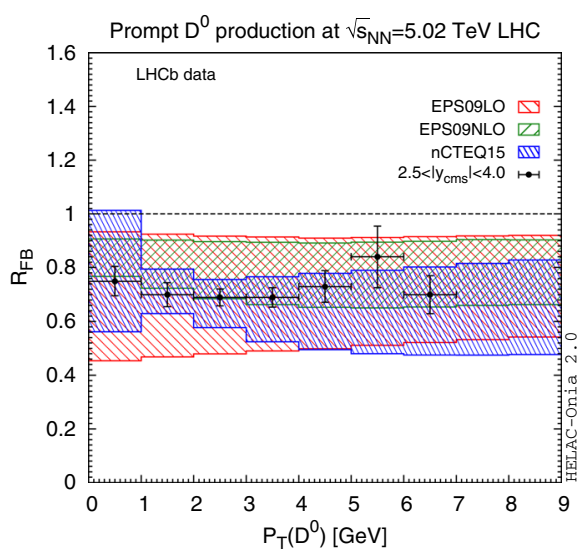

(b)

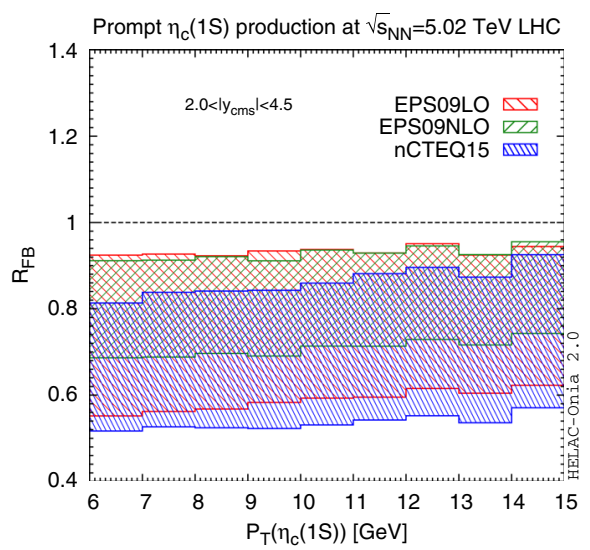

(b)
ALICE, the magnitude of the asymmetry is well compatible with that of nCTEQ15 and EPS09 LO, at the lower edge of the EPS09 NLO range. As for the ATLAS data with a $P_{T}$ cut, their current uncertainties and the reduced magnitude of the effects (since $\left|y_{\text {c.m.s. }}\right|$ is smaller) do not allow for any conclusions.

Figure 14 shows our results for $R_{\mathrm{FB}}$ versus $P_{T}^{J / \psi}$. A clear trend is seen in the LHCb and ALICE results with a ratio increasing with $P_{T}$, starting at 0.6 . $R_{\mathrm{FB}}$ at $P_{T}$ above $10 \mathrm{GeV}$ are compatible with unity, but the larger uncertainties do not exclude values smaller than one. The magnitude of the ratio in the data is compatible with the $3 \mathrm{nPDF}$. More advanced studies are needed to go further in the interpretation of the $P_{T}$ dependence using specific eigensets as opposed to bands. We also recall that a given nPDF set can be compatible with $R_{\mathrm{FB}}$ and not with $R_{p} \mathrm{~Pb}$. This can happen due to specific cancellations in the magnitude of the forward and backward nuclear modifications or to a normalisation offset. In particular, we 
note that there is no tension at all with the ATLAS data for $R_{\mathrm{FB}}$ (Figs. 13c, 14c) unlike the case of $R_{p \mathrm{~Pb}}$ (Figs. 8c, 9b). We are inclined to attribute this to a normalisation offset from the $p p$ baseline whose effect disappears in $R_{\mathrm{FB}}$.

We have also computed $R_{\mathrm{FB}}$ for $\Upsilon(1 S)$ (Fig. 15) and $D^{0}$ (Fig. 16). The same remarks as for the $J / \psi$ case apply. No tension between the data and our computation are found. Just as for the ATLAS $J / \psi$ data, the good agreement with the $D^{0}$ LHCb data may indicate that a slight offset in the normalisation affects $R_{p} \mathrm{~Pb}$ as plotted on Fig. 11 . Whereas the $R_{p} \mathrm{~Pb}$ values point at a smaller suppression than those encoded in the nPDFs (in particular nCTEQ15), the magnitude of $R_{\mathrm{FB}}$ is very well accounted by $\mathrm{nCTEQ} 15$ and corresponds to the strongest magnitude encoded in EPS09 NLO. For completeness, we have also computed $R_{\mathrm{FB}}$ of prompt $\eta_{c}(1 S)$ (see Fig. 17).

\subsection{A few words on the (factorisation) scale dependence}

As our results have shown, the nPDF uncertainties are significant and, in most cases, larger than those of the data. Moreover, the uncertainties of different nPDF sets do not necessarily overlap. Adding results obtained with the DSSZ set [26] would even probably enlarge the spread of the results. This is a strong motivation to learn to which extent these data could be used in the future to constrain the nPDFs. We are hopeful that our simple procedure can be an useful tool along this agenda.

Yet, as emphasised in [30], one should not forget one additional piece of uncertainty inherent to the use of nPDFs and the collinear factorisation, namely the (factorisation) scale, $\mu_{F}$, at which the parton densities should be evaluated. It is customary to pick up a natural scale related to the process (mass of the produced particle, typical momentum exchange, ...) and to vary it by a factor two, or so, about this natural value. It happens that the magnitude of the nuclear effects encoded in the nPDFs do depend on $\mu_{F}$ and we are not aware of strong theoretical arguments allowing one to bypass these considerations.

Under our procedure, there is absolutely no difficulty to evaluate the additional uncertainty attached to the unknown value of $\mu_{F}{ }^{7}$ Owing to our parametrisation of the $p p$ data, the $\mu_{F}$ dependence in the proton PDFs mostly cancels in the fit. If we were to choose a different value of $\mu_{F}$ at which we evaluate the proton PDFs and refit the $p p$ data, the denominator of $R_{p \mathrm{~Pb}}$ would nearly be similar.

In Fig. 18, we thus only show the variation of $R_{p} \mathrm{~Pb}$ due to the change of $\mu_{F}$ in the evaluation of the nuclear PDFs, or in the ratio $R_{g}$ for EPS09. It is interesting - but not surprising - to note that both nPDF and $\mu_{F}$ uncertainties depend

\footnotetext{
7 In fact, the evaluation of this uncertainty can be automatised as for that of the nPDFs.
}

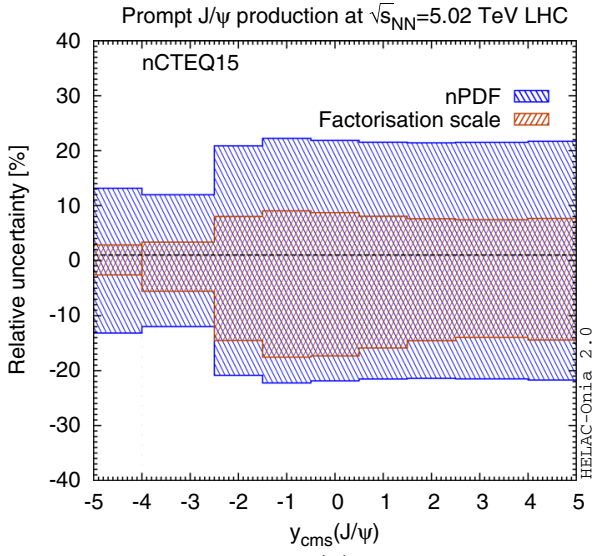

(a)

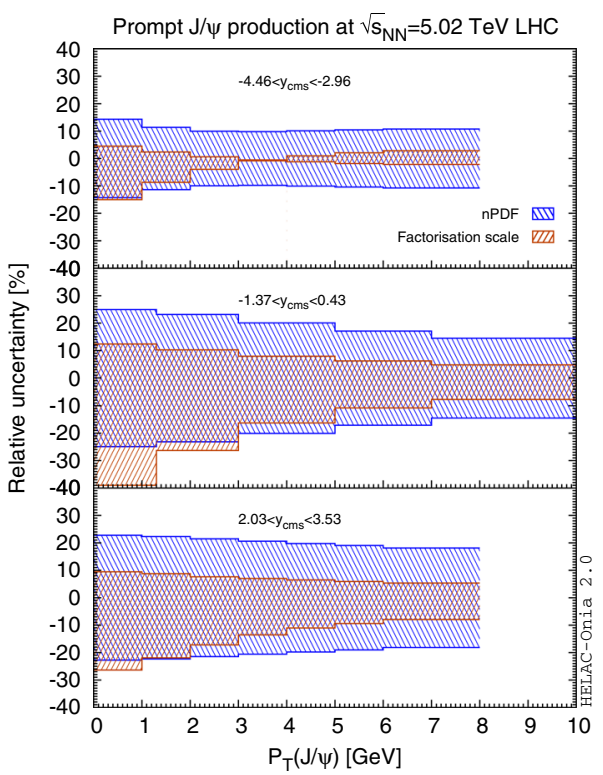

(b)

Fig. 18 Comparison between the nPDF and the $\mu_{F}$ relative uncertainties on $R_{p \mathrm{~Pb}}$ as a function of rapidity (a) and transverse-momentum (b) for prompt $J / \psi$ production in $p$ Pbcollisions at $\sqrt{s_{N N}}=5.02 \mathrm{TeV}$

on $y$ and $P_{T}$. For $y$, it is obvious since it is connected to $x_{1}$ and $x_{2}$. The $\mu_{F}$ dependence is more marked at low $P_{T}$ which is also not a surprise. Overall, even though it is nearly everywhere smaller that the nPDF uncertainties (of a given set), the scale uncertainty is significant and should be kept in mind when comes the time for more quantitative theory-data comparisons.

\section{Conclusions}

We have devised a model-independent procedure to evaluate the impact of the nuclear modification of the gluon densities on hard probes produced in proton-nucleus collisions at colliders energies. It is particularly tailored for two-to-two par- 
tonic scatterings, relevant for quarkonium and heavy-meson production. The model independence of our procedure lies in the parametrisation of the partonic amplitude squared with parameters fit to $p p$ collision data in similar kinematical conditions as the $p \mathrm{~Pb}$ data to be described; this is in contrast to other tools based on matrix elements calculated at a fixed order in perturbative theory.

We have illustrated the capabilities of our approach by computing the cross sections as well as the nuclear modifications factor for $J / \psi, \Upsilon$ and $D^{0}$ production at the LHC. Even though our objective was not to argue that the nPDF effect is the dominant one in this energy range, we have not found out any significant tension between our computations using three common nPDFs (EPS09 LO \& NLO and nCTEQ15) and the existing data. To further highlight the potentialities of the approach, we have made predictions for $\eta_{c}$ production which might be at reach for the LHCb collaboration. We have also made predictions for the $8 \mathrm{TeV} p \mathrm{~Pb}$ run (see the Appendix).

As outlooks for physics studies, our method can easily be transposed to $B$ hadron production. It should also be possible to apply it for non-prompt charmonia provided that the kinematical shift between the $b$-quark and the charmonium is correctly accounted for. On the side of the tool itself, ${ }^{8}$ we plan to improve it such that it could automatically provide the user with the nuclear modification factors starting for measured $p p$ data.
Acknowledgements We are very grateful to R. Arnaldi, E.G. Ferreiro, F. Fleuret, C. Hadjidakis, D. Kikola, Y. Kim, A. Kusina, S. Lee, A. Rakatozafindrabe, C. Salgado, I. Schienbein, R. Vogt, Z. Yang, Y. Zhang for stimulating discussions. The work of J.P.L. is supported in part by the French CNRS via the LIA FCPPL (Quarkonium4AFTER) and the Défi Inphyniti-Théorie LHC France. H.S.S. is supported by the ERC Grant 291377 LHCtheory Theoretical predictions and analyses of LHC physics: advancing the precision frontier

Open Access This article is distributed under the terms of the Creative Commons Attribution 4.0 International License (http://creativecomm ons.org/licenses/by/4.0/), which permits unrestricted use, distribution, and reproduction in any medium, provided you give appropriate credit to the original author(s) and the source, provide a link to the Creative Commons license, and indicate if changes were made.

Funded by $\mathrm{SCOAP}^{3}$.

\section{Appendix: Predictions for the $p \mathrm{~Pb}$ run at $8 \mathrm{TeV}$}

In this appendix, we show predictions for the $p \mathrm{~Pb}$ run at $8 \mathrm{TeV}$ using the foreseen experimental acceptance of the ALICE, ATLAS, CMS and LHCb experiments. These can serve as baselines to analyse whether nuclear effects beyond those encapsulated in the nPDF are visible at the highest energy possible for proton-nucleus at the LHC (Fig. 19).

\footnotetext{
8 The code can be downloaded at http://helac-phegas.web.cern.ch/ helac-phegas/helac-onia.html.
} 


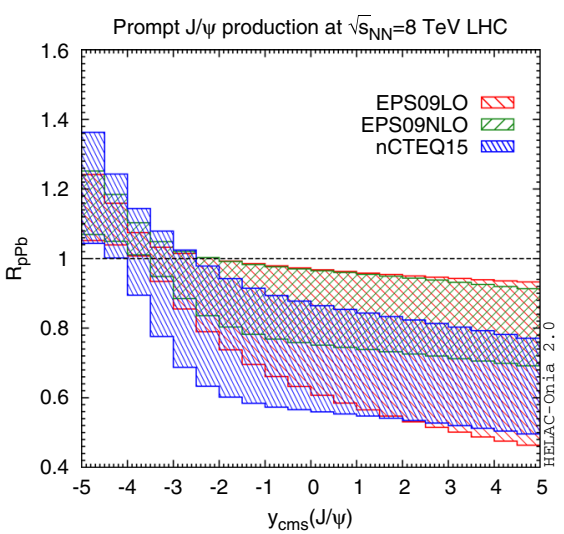

(a)

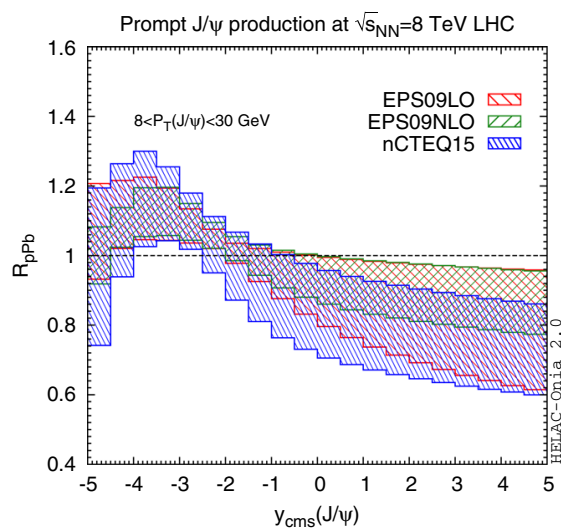

(b)

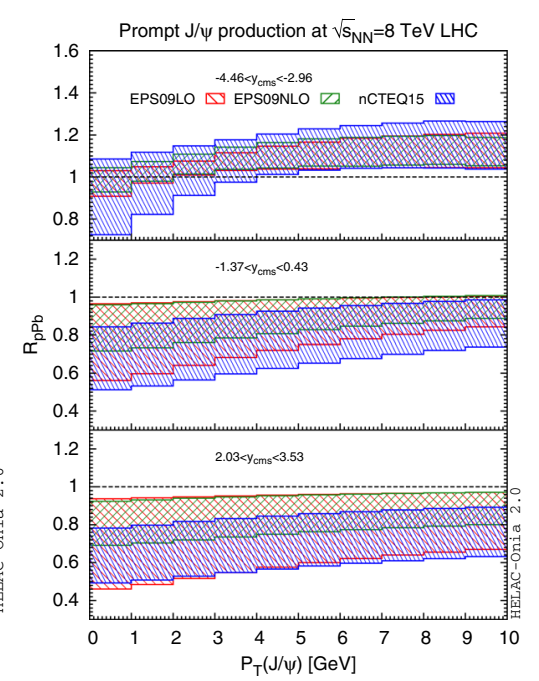

(c)

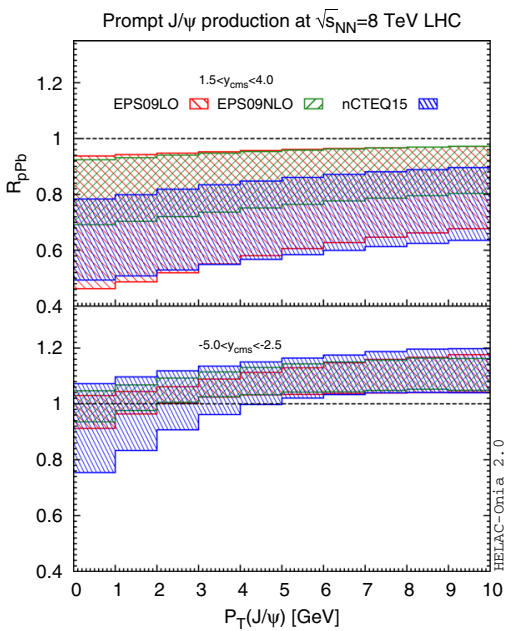

(d)

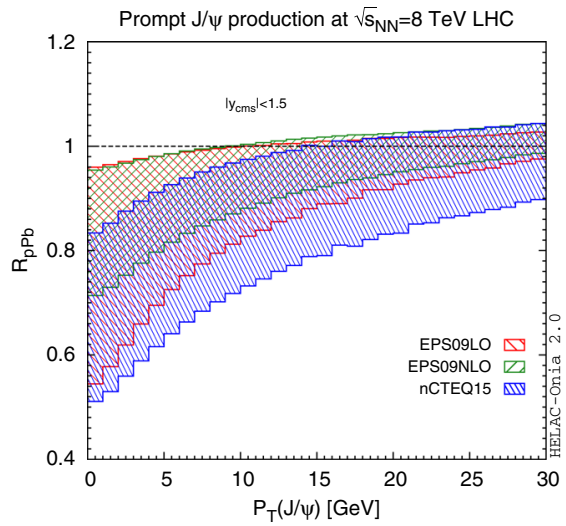

(e)

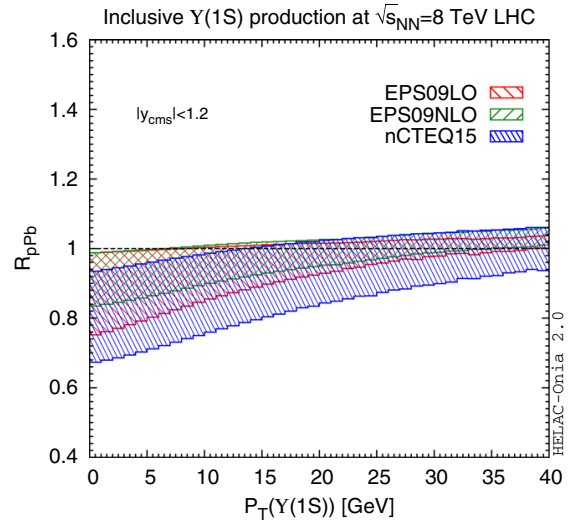

(f)

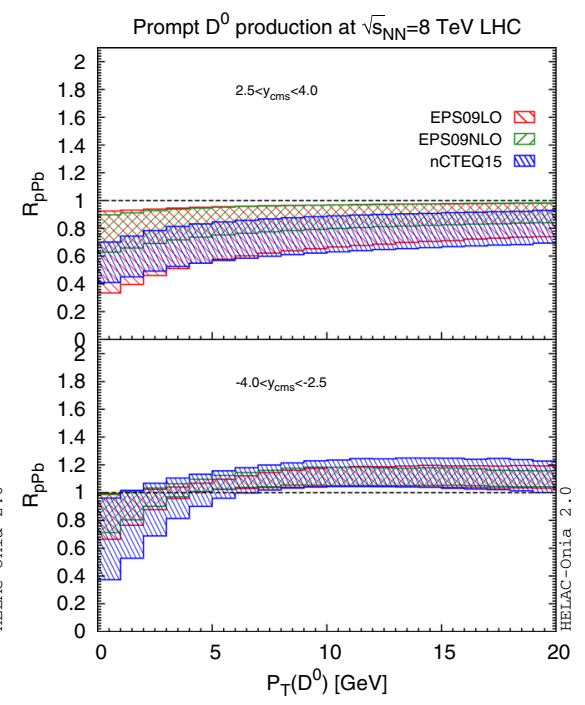

(i)

Fig. 19 Predictions for $8 \mathrm{TeV}$ in different rapidity and transverse momentum regions 


\section{References}

1. A. Andronic et al., Heavy-flavour and quarkonium production in the LHC era: from proton-proton to heavy-ion collisions. Eur. Phys. J. C 76(3), 107 (2016). doi:10.1140/epjc/s10052-015-3819-5. arXiv:1506.03981 [nucl-ex]

2. J.P. Lansberg, Theory status of quarkonium production in protonnucleus collisions. J. Phys. Conf. Ser. 668(1), 012019 (2016). doi:10.1088/1742-6596/668/1/012019. arXiv:1510.01818 [nuclth]

3. N. Brambilla et al., Heavy quarkonium: progress, puzzles, and opportunities. Eur. Phys. J. C 71, 1534 (2011). doi:10.1140/epjc/ s10052-010-1534-9. arXiv:1010.5827 [hep-ph]

4. R. Rapp, D. Blaschke, P. Crochet, Charmonium and bottomonium production in heavy-ion collisions. Prog. Part. Nucl. Phys. 65, 209266 (2010). doi:10.1016/j.ppnp.2010.07.002. arXiv:0807.2470 [hep-ph]

5. A.D. Frawley, T. Ullrich, R. Vogt, Heavy flavor in heavy-ion collisions at RHIC and RHIC II. Phys. Rept. 462, 125-175 (2008). doi:10.1016/j.physrep.2008.04.002. arXiv:0806.1013 [nucl-ex]

6. J.P. Lansberg, $J / \psi, \psi$ and $\Upsilon$ production at hadron colliders: A review. Int. J. Mod. Phys. A 21, 3857-3916 (2006). doi:10.1142/ S0217751X06033180. arXiv:hep-ph/0602091 [hep-ph]

7. C. Gerschel, J. Hufner, A contribution to the suppression of the J/psi Meson produced in high-energy nucleus nucleus collisions. Phys. Lett. B 207, 253-256 (1988). doi:10.1016/0370-2693(88)90570-9

8. R. Vogt, $J / \psi$ production and suppression. Phys. Rept. 310, 197260 (1999). doi:10.1016/S0370-1573(98)00074-X

9. E.G. Ferreiro, Excited charmonium suppression in protonnucleus collisions as a consequence of comovers. Phys. Lett. B 749, 98 103 (2015). doi:10.1016/j.physletb.2015.07.066. arXiv:1411.0549 [hep-ph]

10. A. Capella, E.G. Ferreiro, J/psi suppression at $\mathrm{s}^{* *}(1 / 2)=200-$ $\mathrm{GeV}$ in the comovers interaction model. Eur. Phys. J. C 42, 419-424 (2005). doi:10.1140/epjc/s2005-02348-0. arXiv:hep-ph/0505032 [hep-ph]

11. A. Capella, E.G. Ferreiro, A.B. Kaidalov, Nonsaturation of the J/psi suppression at large transverse energy in the comovers approach. Phys. Rev. Lett. 85, 2080-2083 (2000). doi:10.1103/PhysRevLett. 85.2080. arXiv:hep-ph/0002300 [hep-ph]

12. S. Gavin, R. Vogt, $J / \psi$ suppression from Hadron-Nucleus to nucleus-nucleus collisions. Nucl. Phys. B 345, 104-124 (1990). doi:10.1016/0550-3213(90)90610-P

13. F. Arleo, S. Peigne, J/ $\psi$ suppression in $\mathrm{p}-\mathrm{A}$ collisions from parton energy loss in cold QCD matter. Phys. Rev. Lett. 109, 122301 (2012). doi:10.1103/PhysRevLett.109.122301. arXiv:1204.4609 [hep-ph]

14. R. Sharma, I. Vitev, High transverse momentum quarkonium production and dissociation in heavy ion collisions. Phys. Rev. C 87(4), 044905 (2013). doi:10.1103/PhysRevC.87.044905. arXiv:1203.0329 [hep-ph]

15. F. Arleo, S. Peigne, T. Sami, Revisiting scaling properties of medium-induced gluon radiation. Phys. Rev. D 83, 114036 (2011). doi:10.1103/PhysRevD.83.114036. arXiv:1006.0818 [hep-ph]

16. S.J. Brodsky, P. Hoyer, A bound on the energy loss of partons in nuclei. Phys. Lett. B 298, 165-170 (1993). doi:10.1016/ 0370-2693(93)91724-2. arXiv:hep-ph/9210262 [hep-ph]

17. S. Gavin, J. Milana, Energy loss at large $\mathrm{x}(\mathrm{F})$ in nuclear collisions. Phys. Rev. Lett. 68, 1834-1837 (1992). doi:10.1103/PhysRevLett. 68.1834

18. S.J. Brodsky, P. Hoyer, The Nucleus as a Color Filter in QCD Decays: Hadroproduction in Nuclei. Phys. Rev. Lett. 63, 1566 (1989). doi:10.1103/PhysRevLett.63.1566

19. B. Ducloué, T. Lappi, H. Mantysaari, Forward $J / \psi$ production in proton-nucleus collisions at high energy. Phys. Rev.
D 91(11), 114005 (2015). doi:10.1103/PhysRevD.91.114005. arXiv:1503.02789 [hep-ph]

20. Y.-Q. Ma, R. Venugopalan, H.-F. Zhang, $J / \psi$ production and suppression in high energy proton-nucleus collisions. Phys. Rev. D 92, 071901 (2015). doi:10.1103/PhysRevD.92.071901. arXiv:1503.07772 [hep-ph]

21. H. Fujii, K. Watanabe, Heavy quark pair production in high energy pA collisions: Quarkonium. Nucl. Phys. A 915, 1-23 (2013). doi:10.1016/j.nuclphysa.2013.06.011. arXiv:1304.2221 [hep-ph]

22. J.-W. Qiu, P. Sun, B.-W. Xiao, F. Yuan, Universal suppression of heavy quarkonium production in pA collisions at low transverse momentum. Phys. Rev. D 89(3), 034007 (2014). doi:10.1103/ PhysRevD.89.034007. arXiv:1310.2230 [hep-ph]

23. B. Kopeliovich, A. Tarasov, J. Hufner, Coherence phenomena in charmonium production off nuclei at the energies of RHIC and LHC. Nucl. Phys. A 696, 669-714 (2001). doi:10.1016/ S0375-9474(01)01220-9. arXiv:hep-ph/0104256 [hep-ph]

24. K. Kovarik et al., nCTEQ15-Global analysis of nuclear parton distributions with uncertainties in the CTEQ framework. Phys. Rev. D 93(8), 085037 (2016). doi:10.1103/PhysRevD.93.085037. arXiv:1509.00792 [hep-ph]

25. J.F. Owens, A. Accardi, W. Melnitchouk, Global parton distributions with nuclear and finite- $Q^{2}$ corrections. Phys. Rev. D 87(9), 094012 (2013). doi:10.1103/PhysRevD.87.094012. arXiv:1212.1702 [hep-ph]

26. D. de Florian, R. Sassot, P. Zurita, M. Stratmann, Global analysis of nuclear parton distributions. Phys. Rev. D 85, 074028 (2012). doi:10.1103/PhysRevD.85.074028. arXiv:1112.6324 [hep-ph]

27. K.J. Eskola, H. Paukkunen, C.A. Salgado, EPS09: A new generation of NLO and LO nuclear parton distribution functions. JHEP 04, 065 (2009). doi:10.1088/1126-6708/2009/04/065. arXiv:0902.4154 [hep-ph]

28. M. Hirai, S. Kumano, T.H. Nagai, Determination of nuclear parton distribution functions and their uncertainties in next-to-leading order. Phys. Rev. C 76, 065207 (2007). doi:10.1103/PhysRevC.76. 065207. arXiv:0709.3038 [hep-ph]

29. J.L. Albacete et al., Predictions for $p+\mathrm{Pb}$ Collisions at $\sqrt{s_{N N}}=5 \mathrm{TeV}$ : Comparison with data. Int. J. Mod. Phys. E 25(09), 1630005 (2016). doi:10.1142/S0218301316300058. arXiv:1605.09479 [hep-ph]

30. E.G. Ferreiro, F. Fleuret, J.P. Lansberg, A. Rakotozafindrabe, Impact of the nuclear modification of the gluon densities on $J / \psi$ production in $p \mathrm{~Pb}$ collisions at $\sqrt{s_{N N}}=5 \mathrm{TeV}$. Phys. Rev. C 88(4), 047901 (2013). doi:10.1103/PhysRevC.88.047901. arXiv:1305.4569 [hep-ph]

31. R. Gauld, J. Rojo, Precision determination of the small- $x$ gluon from charm production at LHCb. arXiv:1610.09373 [hep-ph]

32. R. Vogt, Shadowing and absorption effects on $\mathrm{J} / \mathrm{psi}$ production in dA collisions. Phys. Rev. C 71, 054902 (2005). doi:10.1103/ PhysRevC.71.054902. arXiv:hep-ph/0411378 [hep-ph]

33. F. Arleo, V.-N. Tram, A Systematic study of J/psi suppression in cold nuclear matter. Eur. Phys. J. C 55, 449-461 (2008). doi:10. 1140/epjc/s10052-008-0604-8. arXiv:hep-ph/0612043 [hep-ph]

34. F. Arleo, Constraints on nuclear gluon densities from J/psi data. Phys. Lett. B 666, 31-33 (2008). doi:10.1016/j.physletb.2008.06. 074. arXiv:0804.2802 [hep-ph]

35. C. Lourenco, R. Vogt, H.K. Woehri, Energy dependence of J/psi absorption in proton-nucleus collisions. JHEP 02, 014 (2009). doi:10.1088/1126-6708/2009/02/014. arXiv:0901.3054 [hep-ph]

36. R. Vogt, Cold nuclear matter effects on $J / \psi$ and $\Upsilon$ production at the LHC. Phys. Rev. C 81, 044903 (2010). doi:10.1103/PhysRevC. 81.044903. arXiv: 1003.3497 [hep-ph]

37. E.G. Ferreiro, F. Fleuret, A. Rakotozafindrabe, Transverse momentum dependence of $\mathrm{J} / \mathrm{psi}$ shadowing effects. Eur. Phys. J. C 61, 859-864 (2009). doi:10.1140/epjc/s10052-008-0843-8. arXiv:0801.4949 [hep-ph] 
38. E.G. Ferreiro, F. Fleuret, J.P. Lansberg, A. Rakotozafindrabe, Cold nuclear matter effects on $\mathrm{J} / \mathrm{psi}$ production: Intrinsic and extrinsic transverse momentum effects. Phys. Lett. B 680, 50-55 (2009). doi:10.1016/j.physletb.2009.07.076. arXiv:0809.4684 [hep-ph]

39. E.G. Ferreiro, F. Fleuret, J.P. Lansberg, A. Rakotozafindrabe, Centrality, rapidity and transverse-momentum dependence of cold nuclear matter Effects on J/Psi production in $\mathrm{d} \mathrm{Au}, \mathrm{Cu} \mathrm{Cu}$ and $\mathrm{Au} \mathrm{Au}$ collisions at $\mathrm{s}(\mathrm{NN}) * *(1 / 2)=200-\mathrm{GeV}$. Phys. Rev. C 81, 064911 (2010). doi:10.1103/PhysRevC.81.064911. arXiv:0912.4498 [hep-ph]

40. Z. Conesa del Valle, E.G. Ferreiro, F. Fleuret, J.P. Lansberg, A. Rakotozafindrabe, Open-beauty production in $p \mathrm{~Pb}$ collisions at $\sqrt{s_{N N}}=5 \mathrm{TeV}$ : effect of the gluon nuclear densities. arXiv:1402.1747 [hep-ph] [Nucl. Phys. A 926, 236 (2014)]

41. T. Carli, D. Clements, A. Cooper-Sarkar, C. Gwenlan, G.P. Salam, F. Siegert, P. Starovoitov, M. Sutton, A posteriori inclusion of parton density functions in NLO QCD final-state calculations at hadron colliders: The APPLGRID Project. Eur. Phys. J. C 66, 503-524 (2010). doi:10.1140/epjc/s10052-010-1255-0. arXiv:0911.2985 [hep-ph]

42. T. Kluge, K. Rabbertz, M. Wobisch, FastNLO: Fast pQCD calculations for PDF fits. in Deep inelastic scattering. Proceedings, 14th International Workshop, DIS 2006, Tsukuba, Japan, April 20-24, 2006, pp. 483-486 (2006). arXiv:hep-ph/0609285 [hep-ph]. http:// 1ss.fnal.gov/cgi-bin/find_paper.pl?conf-06-352

43. H.-S. Shao, HELAC-Onia: An automatic matrix element generator for heavy quarkonium physics. Comput. Phys. Commun. 184, 2562-2570 (2013). doi:10.1016/j.cpc.2013.05.023. arXiv:1212.5293 [hep-ph]

44. H.-S. Shao, HELAC-Onia 2.0: an upgraded matrix-element and event generator for heavy quarkonium physics. Comput. Phys. Commun. 198, 238-259 (2016). doi:10.1016/j.cpc.2015.09.011. arXiv:1507.03435 [hep-ph]

45. M.R. Whalley, D. Bourilkov, R.C. Group, The Les Houches accord PDFs (LHAPDF) and LHAGLUE, in HERA and the LHC: A Workshop on the implications of HERA for LHC physics. Proceedings, Part B (2005). arXiv:hep-ph/0508110 [hep-ph]

46. D. Bourilkov, R.C. Group, M.R. Whalley, LHAPDF: PDF use from the Tevatron to the LHC, in TeV4LHC Workshop4th meeting Batavia, Illinois, October 20-22, 2005 (2006). arXiv:hep-ph/0605240 [hep-ph]

47. A. Buckley, J. Ferrando, S. Lloyd, K. Nordstrm, B. Page, M. Rfenacht, M. Schnherr, G. Watt, LHAPDF6: parton density access in the LHC precision era. Eur. Phys. J. C 75, 132 (2015). doi:10.1140/ epjc/s10052-015-3318-8. arXiv:1412.7420 [hep-ph]

48. C.H. Kom, A. Kulesza, W.J. Stirling, Pair Production of J/psi as a Probe of Double Parton Scattering at LHCb. Phys. Rev. Lett. 107, 082002 (2011). doi:10.1103/PhysRevLett.107.082002. arXiv:1105.4186 [hep-ph]

49. J.-P. Lansberg, H.-S. Shao, $J / \psi$-pair production at large momenta: Indications for double parton scatterings and large $\alpha_{s}^{5}$ contributions. Phys. Lett. B 751, 479-486 (2015). doi:10.1016/j.physletb.2015. 10.083. arXiv:1410.8822 [hep-ph]

50. J.-P. Lansberg, H.-S. Shao, Double-quarkonium production at a fixed-target experiment at the LHC (AFTER@LHC). Nucl. Phys. B 900, 273-294 (2015). doi:10.1016/j.nuclphysb.2015.09. 005. arXiv:1504.06531 [hep-ph]

51. H.-S. Shao, Y.-J. Zhang, Complete study of hadroproduction of a $\Upsilon$ meson associated with a prompt $J / \psi$. Phys. Rev. Lett. 117(6), 062001 (2016). doi:10.1103/PhysRevLett.117.062001. arXiv:1605.03061 [hep-ph]

52. C. Borschensky, A. Kulesza, Double parton scattering in pairproduction of $J / \psi$ mesons at the LHC revisited. arXiv:1610.00666 [hep-ph]

53. S. Dulat, T.-J. Hou, J. Gao, M. Guzzi, J. Huston, P. Nadolsky, J. Pumplin, C. Schmidt, D. Stump, C.P. Yuan, New parton distribu- tion functions from a global analysis of quantum chromodynamics. Phys. Rev. D 93(3), 033006 (2016). doi:10.1103/PhysRevD. 93.033006. arXiv:1506.07443 [hep-ph]

54. H.-L. Lai, M. Guzzi, J. Huston, Z. Li, P.M. Nadolsky, J. Pumplin, C.P. Yuan, New parton distributions for collider physics. Phys. Rev. D 82, 074024 (2010). doi:10.1103/PhysRevD.82.074024. arXiv:1007.2241 [hep-ph]

55. LHCb Collaboration, R. Aaij et al., Measurement of $J / \psi$ production in $p p$ collisions at $\sqrt{s}=7 \mathrm{TeV}$. Eur. Phys. J. C 71, 1645 (2011). doi:10.1140/epjc/s10052-011-1645-y. arXiv:1103.0423 [hep-ex]

56. LHCb Collaboration, R. Aaij et al., Production of J/psi and Upsilon mesons in pp collisions at sqrt(s) $=8 \mathrm{TeV}$. JHEP 06, 064 (2013). doi:10.1007/JHEP06(2013)064. arXiv:1304.6977 [hep-ex]

57. ATLAS Collaboration, G. Aad et al., Measurement of the differential cross-sections of prompt and non-prompt production of $J / \psi$ and $\psi(2 S)$ in $p p$ collisions at $\sqrt{s}=7$ and $8-\mathrm{TeV}$ with the ATLAS detector. Eur. Phys. J. C 76(5), 283 (2016). doi:10.1140/ epjc/s10052-016-4050-8. arXiv:1512.03657 [hep-ex]

58. CMS Collaboration, V. Khachatryan et al., Measurement of $J / \psi$ and (2S) Prompt Double-Differential Cross Sections in pp Collisions at $\sqrt{s}=7$ TeV. Phys. Rev. Lett. 114(19), 191802 (2015). doi:10.1103/PhysRevLett.114.191802. arXiv:1502.04155 [hep-ex]

59. ALICE Collaboration, B.B. Abelev et al., Measurement of quarkonium production at forward rapidity in $p p$ collisions at $\sqrt{s}=$ 7 TeV. Eur. Phys. J. C 74(8), 2974 (2014). doi:10.1140/epjc/ s10052-014-2974-4. arXiv:1403.3648 [nucl-ex]

60. CMS Collaboration, S. Chatrchyan et al., Measurement of the $\Upsilon(1 S), \Upsilon(2 S)$, and $\Upsilon(3 S)$ cross sections in pp collisions at $\sqrt{s}=$ 7 TeV. Phys. Lett. B 727, 101-125 (2013). doi:10.1016/j.physletb. 2013.10.033. arXiv:1303.5900 [hep-ex]

61. LHCb Collaboration, R. Aaij et al., Measurement of Upsilon production in pp collisions at $\sqrt{s}=7$ TeV. Eur. Phys. J. C 72, 2025 (2012). doi:10.1140/epjc/s10052-012-2025-y. arXiv:1202.6579 [hep-ex]

62. LHCb Collaboration, R. Aaij et al., Measurement of the $\eta_{c}(1 S)$ production cross-section in proton-proton collisions via the decay $\eta_{c}(1 S) \rightarrow p \bar{p}$. Eur. Phys. J. C 75(7), 311 (2015). doi:10.1140/ epjc/s10052-015-3502-x. arXiv:1409.3612 [hep-ex]

63. LHCb Collaboration, R. Aaij et al., Prompt charm production in pp collisions at sqrt(s) $=7 \mathrm{TeV}$. Nucl. Phys. B 871 1-20, doi:10. 1016/j.nuclphysb.2013.02.010. arXiv:1302.2864 [hep-ex]

64. LHCb Collaboration, R. Aaij et al., Forward production of $\Upsilon$ mesons in $p p$ collisions at $\sqrt{s}=7$ and 8TeV,. JHEP 11, 103 (2015). doi:10.1007/JHEP11(2015)103. arXiv:1509.02372 [hep-ex]

65. ATLAS Collaboration, G. Aad et al., Measurement of Upsilon production in $7 \mathrm{TeV}$ pp collisions at ATLAS. Phys. Rev. D 87(5), 052004 (2013). doi:10.1103/PhysRevD.87.052004. arXiv:1211.7255 [hep-ex]

66. LHCb Collaboration, R. Aaij et al., Study of $J / \psi$ production and cold nuclear matter effects in $p P b$ collisions at $\sqrt{s_{N N}}=$ 5 TeV. JHEP 02, 072 (2014). doi:10.1007/JHEP02(2014)072. arXiv:1308.6729 [nucl-ex]

67. ALICE Collaboration, J. Adam et al., Rapidity and transversemomentum dependence of the inclusive $\mathrm{J} / \psi$ nuclear modification factor in $\mathrm{p}-\mathrm{Pb}$ collisions at $\sqrt{s_{N N}}=5.02 \mathrm{TeV}$. JHEP 06, 055 (2015). doi:10.1007/JHEP06(2015)055. arXiv:1503.07179 [nuclex]

68. ATLAS Collaboration, G. Aad et al., Measurement of differential $J / \psi$ production cross sections and forward-backward ratios in $\mathrm{p}+\mathrm{Pb}$ collisions with the ATLAS detector. Phys. Rev. C 92(3), 034904 (2015). doi:10.1103/PhysRevC.92.034904. arXiv:1505.08141 [hep-ex]

69. ALICE Collaboration, B.B. Abelev et al., $J / \psi$ production and nuclear effects in p-Pb collisions at $\sqrt{S_{N N}}=5.02 \mathrm{TeV}$. JHEP 02, 
073 (2014). doi:10.1007/JHEP02(2014)073. arXiv:1308.6726 [nucl-ex]

70. LHCb Collaboration, R. Aaij et al., Study of $\Upsilon$ production and cold nuclear matter effects in $p \mathrm{~Pb}$ collisions at $\sqrt{s_{N N}}=5 \mathrm{TeV}$. JHEP07 (2014) 094, doi:10.1007/JHEP07(2014)094. arXiv:1405.5152 [nucl-ex]

71. ALICE Collaboration, B.B. Abelev et al., Production of inclusive $\Upsilon(1 \mathrm{~S})$ and $\Upsilon(2 \mathrm{~S})$ in $\mathrm{p}-\mathrm{Pb}$ collisions at $\sqrt{\mathbf{s}_{\mathrm{NN}}}=\mathbf{5 . 0 2} \mathrm{TeV}$. Phys. Lett. B 740 105-117 (2015). doi:10.1016/j.physletb.2014.11.041. arXiv:1410.2234 [nucl-ex]

72. ATLAS Collaboration, Measurement of $\Upsilon(\mathrm{nS})$ production with $p+P b$ collisions at $\sqrt{s_{\mathrm{NN}}}=5.02 \mathrm{TeV}$ and pp collisions at $\sqrt{s}=$ 2.76 TeV, Tech. Rep. ATLAS-CONF-2015-050 (CERN, Geneva, 2015) https://cds.cern.ch/record/2055266
73. LHCb Collaboration, Study of cold nuclear matter effects using prompt $D^{0}$ meson production in $\mathrm{pPb}$ collisions at LHCb, Tech. Rep. LHCb-CONF-2016-003 (CERN, Geneva, 2016). http://cds. cern.ch/record/2138946

74. ALICE Collaboration, B.B. Abelev et al., Measurement of prompt $D$-meson production in $p-P b$ collisions at $\sqrt{s_{N N}}=5.02$ TeV. Phys. Rev. Lett. 113(23), 232301 (2014). doi:10.1103/ PhysRevLett.113.232301. arXiv:1405.3452 [nucl-ex]

75. ATLAS Collaboration, T.A. Collaboration, Study of $J / \psi$ and $\psi(2 \mathrm{~S})$ production in $\sqrt{s_{\mathrm{NN}}}=5.02 \mathrm{TeV} p+\mathrm{Pb}$ and $\sqrt{s}=2.76 \mathrm{TeV}$ pp collisions with the ATLAS detector, Tech. Rep. ATLAS-CONF2015-023 (2015) 